Structured Mapping Problems for Matrices Associated with Scalar Products Part I: Lie and Jordan Algebras

Mackey, D. Steven and Mackey, Niloufer and Tisseur, Françoise

2006

MIMS EPrint: 2006.44

Manchester Institute for Mathematical Sciences

School of Mathematics

The University of Manchester

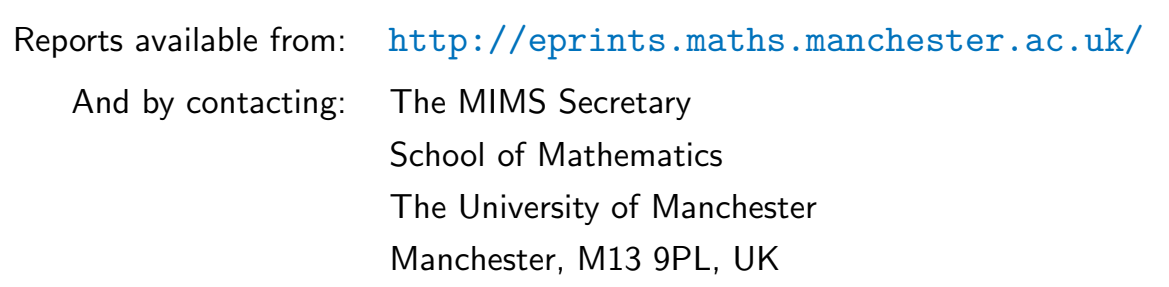




\title{
STRUCTURED MAPPING PROBLEMS FOR MATRICES ASSOCIATED WITH SCALAR PRODUCTS PART I: LIE AND JORDAN ALGEBRAS*
}

\author{
D. STEVEN MACKEY ${ }^{\dagger}$, NILOUFER MACKEY ${ }^{\ddagger}$, AND FRANÇOISE TISSEUR*
}

\begin{abstract}
Given a class of structured matrices $\mathbb{S}$, we identify pairs of vectors $x, b$ for which there exists a matrix $A \in \mathbb{S}$ such that $A x=b$, and also characterize the set of all matrices $A \in \mathbb{S}$ mapping $x$ to $b$. The structured classes we consider are the Lie and Jordan algebras associated with orthosymmetric scalar products. These include (skew-)symmetric, (skew-)Hamiltonian, pseudo (skew-)Hermitian, persymmetric and perskew-symmetric matrices. Structured mappings with extremal properties are also investigated. In particular, structured mappings of minimal rank are identified and shown to be unique when rank-1 is achieved. The structured mapping of minimal Frobenius norm is always unique and explicit formulas for it and its norm are obtained. Finally the set of all structured mappings of minimal 2-norm is characterized. Our results generalize and unify existing work, answer a number of open questions, and provide useful tools for structured backward error investigations.
\end{abstract}

Key words. Lie algebra, Jordan algebra, scalar product, bilinear form, sesquilinear form, orthosymmetric, adjoint, structured matrix, backward error, Hamiltonian, skew-Hamiltonian, Hermitian, complex symmetric, skew-symmetric, persymmetric, perskew-symmetric, minimal rank, minimal Frobenius norm, minimal 2-norm.

AMS subject classifications. 15A04, 15A57, 15A60, 15A63, 65F 30, 65F35

1. Introduction. The problem of finding all matrices $A$ that map a given nonzero vector $x \in \mathbb{K}^{n}$ to a given vector $b \in \mathbb{K}^{m}$, where $\mathbb{K}$ is a fixed field, can be solved using elementary means [10]. Trenkler [20] recently revisited this problem, giving a solution using generalized inverses:

$$
A=b x^{\dagger}+Z\left(I_{n}-x x^{\dagger}\right)
$$

where $I_{n}$ is the $n \times n$ identity matrix, $Z \in \mathbb{K}^{m \times n}$ is arbitrary and $x^{\dagger}$ is any generalized inverse of $x$. In this work we restrict the permissible transformations to a class of structured matrices $\mathbb{S} \subset \mathbb{K}^{n \times n}$ and consider the following structured mapping problems:

Existence: For which vectors $x, b$ does there exist some $A \in \mathbb{S}$ such that $A x=b$ ?

Characterization: Determine the set $\mathcal{S}=\{A \in \mathbb{S}: A x=b\}$ of all structured mappings taking $x$ to $b$.

We present a complete, unified solution for these two problems when $\mathbb{S}$ is the Lie or Jordan algebra associated with an orthosymmetric scalar product. These include, for example, symmetric and skew-symmetric, Hermitian, pseudo Hermitian and skewHermitian, Hamiltonian, persymmetric and perskew-symmetric matrices. We will assume that $x \neq 0$ throughout, since both problems have trivial solutions if $x=0$.

Answers to some particular instances of these structured mapping problems can be found in the literature. Liu and Leaker [9, Lem. 1] show that for $x, b \in \mathbb{R}^{n}, x$ can be mapped to $b$ by a real skew-symmetric matrix if and only if $x$ and $b$ are orthogonal.

\footnotetext{
*Version of March 27, 2006.

${ }^{\dagger}$ School of Mathematics, The University of Manchester, Sackville Street, Manchester, M60 1QD, UK (\{smackey,ftisseur\}@ma.man.ac.uk, http://www.ma.man.ac.uk/ ftisseur/). This work was supported by Engineering and Physical Sciences Research Council grant GR/S31693.

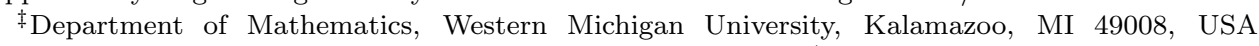
(nil.mackey@wmich.edu, http://homepages.wmich.edu/ ${ }^{\sim m a c k e y /) .}$
} 
Khatri and Mitra [8] and later Sun [16] address the existence and characterization problems for the matrix equation $A X=B$, where $X, B$ are matrices and the unknown $A$ is Hermitian; the skew-Hermitian and complex symmetric cases are covered in [18]. Restricting the results of [8], [16] and [18] to the case when $X$ and $B$ are vectors yields one among the many representations of the set $\mathcal{S}$ identified in this paper. Structured mapping problems for double structures, for structures that do not arise in the context of a scalar product, and for some specific nonlinear structures have also been investigated (see [5], [6], [14], [17] and [21] for examples).

One of our motivations for studying these problems stems from the analysis of structured backward errors in the solutions to structured linear systems and structured eigenproblems [7], [18], [19]. Recall that a backward error of an approximate solution $y$ to a linear system $A x=b$ is a measure of the smallest perturbation $E$ such that $(A+E) y=b$. When $A$ is in some linearly structured class $\mathbb{S}$ one may want to require $E$ to have the same structure; the structured backward error is then a measure of the smallest structured perturbation $E$ such that $E y=r:=b-A y$. Hence solving the structured mapping problem is the first step towards obtaining explicit expressions for structured backward errors.

For any linear matrix structure $\mathbb{S}$ it is possible to obtain a characterization of the structured mapping set $\mathcal{S}$ using the Kronecker product approach described in [4]. The equation $A x=b$ is rewritten as $\left(x^{T} \otimes I_{n}\right) \operatorname{vec}(A)=b$, where $\otimes$ denotes the Kronecker product and vec is the operator that stacks the columns of a matrix into one long vector. The linear nature of the matrix structure is then encoded by $\operatorname{vec}(A)=\Pi_{\mathbb{S}} p$, where $\Pi_{\mathbb{S}}$ is an $n^{2} \times m$ pattern matrix giving (in essence) a basis for the structured class $\mathbb{S}$, and $p$ is an $m$-dimensional vector of parameters $\left(m=\operatorname{dim} \mathbb{S} \leq n^{2}\right)$. Hence

$$
\mathcal{S}=\left\{A \in \mathbb{K}^{n \times n}:\left(x^{T} \otimes I_{n}\right) \Pi_{\mathbb{S}} p=b, \operatorname{vec}(A)=\Pi_{\mathbb{S}} p\right\} .
$$

Note that there may be no solution to the system $\left(x^{T} \otimes I_{n}\right) \Pi_{\mathbb{S}} p=b$ if $\left(x^{T} \otimes I_{n}\right) \Pi_{\mathbb{S}}$ is rank deficient or if the system is overdetermined $(n<m)$. When they exist, solutions can be obtained from the singular value decomposition of $\left(x^{T} \otimes I_{n}\right) \Pi_{\mathbb{S}}$. In particular, if the system is underdetermined and consistent, and if the pattern matrix $\Pi_{\mathbb{S}}$ is chosen so that $\|p\|_{2}=\|A\|_{F}$ for all $A \in \mathbb{S}$ (i.e., $\Pi_{\mathbb{S}}$ contains an orthonormal basis for $\mathbb{S}$ in the Frobenius inner product), then the solution $A \in \mathbb{S}$ with minimal Frobenius norm is given in terms of the pseudo-inverse by $p=\left(\left(x^{T} \otimes I_{n}\right) \Pi_{\mathbb{S}}\right)^{+} b$. As a result we obtain a computable expression for the structured backward error:

$$
\eta_{F}(y)=\min \left\{\|E\|_{F}:(A+E) y=b, E \in \mathbb{S}\right\}=\left\|\left(\left(y^{T} \otimes I_{n}\right) \Pi_{\mathbb{S}}\right)^{+}(b-A y)\right\|_{2}
$$

There are several disadvantages associated with the Kronecker product approach. The existence of structured solutions to $A x=b$ may not be easy to check. In addition, the set $\mathcal{S}$ of all structured mappings is given only implicitly. Also, amongst all solutions in $\mathcal{S}$, it is difficult to distinguish ones with special properties, other than the one of minimal Frobenius norm. The structured backward error expression in (1.2) is expensive to evaluate and difficult to compare with its unstructured counterpart $\|b-A y\|_{2}$.

By contrast, the work presented here gives easy-to-check conditions for the existence problem and an explicit solution for the characterization problem when $\mathbb{S}$ is the Lie or Jordan algebra of a scalar product. The set $\mathcal{S}$ is rewritten as

$$
\mathcal{S}=B+\{A \in \mathbb{S}: A x=0\}
$$


where $B$ is any particular solution of the nonhomogeneous mapping problem. We provide a set of possible particular solutions $B$ for a given class $\mathbb{S}$ and given vectors $x$ and $b$, thus giving multiple ways of representing $\mathcal{S}$. This enables one to more easily identify structured mappings with minimal rank or minimal Frobenius norm and to readily derive bounds for the ratio between the structured and unstructured backward errors [19]. A multiplicative approach, as opposed to the additive approach in (1.3), is used to characterize the set of all minimal 2-norm structured mappings in $\mathcal{S}$. From this characterization, minimal 2-norm mappings of minimal rank and minimal 2-norm mappings of minimal Frobenius norm can be identified.

Here is an illustration of what is obtained by applying our general results to a particular structure $\mathbb{S}$, in this case the Lie algebra of complex skew-symmetric matrices. For given $x, b \in \mathbb{C}^{n}$ our results imply that

$$
\mathcal{S}:=\left\{A \in \mathbb{C}^{n \times n}: A x=b, A^{T}=-A\right\} \text { is nonempty } \Longleftrightarrow x^{T} b=0,
$$

and that

$$
\mathcal{S}=b w^{T}-w^{T} b+\left\{\left(I-v x^{T}\right) L\left(I-x v^{T}\right): L \in \mathbb{C}^{n \times n}, L^{T}=-L\right\},
$$

where $w, v \in \mathbb{C}^{n}$ are any fixed but arbitrary vectors chosen such that $w^{T} x=v^{T} x=1$. All solutions in $\mathcal{S}$ of the form $b w^{T}-w^{T} b$ (corresponding to setting $L=0$ in (1.4)) have minimal rank, and the choice $w=\bar{x} /\|x\|_{2}^{2}, L=0$ gives the unique solution $A_{\mathrm{opt}}$ of minimal Frobenius norm:

$$
A_{\mathrm{opt}}=\left(b \bar{x}^{T}-\bar{x} b^{T}\right) /\|x\|_{2}^{2}, \quad\left\|A_{\mathrm{opt}}\right\|_{F}=\min _{A \in \mathcal{S}}\|A\|_{F}=\sqrt{2}\|b\|_{2} /\|x\|_{2} .
$$

The set $\mathcal{M}=\left\{A \in \mathcal{S}:\|A\|_{2}=\min _{B \in \mathcal{S}}\|B\|_{2}\right\}$ of all minimal 2-norm mappings can be characterized by

$$
\mathcal{M}=\frac{\|b\|_{2}}{\|x\|_{2}}\left\{U^{T} \operatorname{diag}\left(\left[\begin{array}{cc}
0 & -1 \\
1 & 0
\end{array}\right], S\right) U: S \in \mathbb{C}^{(n-2) \times(n-2)}, S^{T}=-S,\|S\|_{2} \leq 1\right\},
$$

where $U^{*}[x \bar{b}]=\left[\|x\|_{2} e_{1}\|b\|_{2} e_{2}\right]$, i.e., $U^{*}$ is the unitary factor of the $\mathrm{QR}$ factorization of $[x \bar{b}]$ with $R$ forced to have positive entries. For this structure $\mathbb{S}$ it turns out that $A_{\text {opt }} \in \mathcal{M}$, so $A_{\text {opt }}$ is a solution of minimal rank, minimal Frobenius norm and minimal 2-norm. As a consequence of (1.5) an explicit formula for the structured backward error in (1.2) for this class $\mathbb{S}$ is given for the Frobenius norm by

$$
\eta_{F}(y)=\sqrt{2} \frac{\|A y-b\|_{2}}{\|y\|_{2}},
$$

which is immediately seen to differ from its unstructured counterpart by a factor of only $\sqrt{2}$. For the 2 -norm the structured and unstructured backward errors are equal.

In summary, the results here generalize and unify existing work, answer a number of open questions, and provide useful tools for the investigation of structured backward errors. After some preliminaries in section 2, a complete solution to the existence and characterization problems is presented in sections 3 and 4 . In section 5 we identify structured solutions of minimal rank, minimal Frobenius norm and minimal 2-norm, and investigate their uniqueness.

\section{Preliminaries.}


2.1. Scalar products. A bilinear form on $\mathbb{K}^{n}(\mathbb{K}=\mathbb{R}, \mathbb{C})$ is a map $(x, y) \mapsto$ $\langle x, y\rangle$ from $\mathbb{K}^{n} \times \mathbb{K}^{n}$ to $\mathbb{K}$, which is linear in each argument. If $\mathbb{K}=\mathbb{C}$, the map $(x, y) \mapsto\langle x, y\rangle$ is a sesquilinear form if it is conjugate linear in the first argument and linear in the second. To a bilinear form on $\mathbb{K}^{n}$ is associated a unique $M \in \mathbb{K}^{n \times n}$ such that $\langle x, y\rangle=x^{T} M y$ for all $x, y \in \mathbb{K}^{n}$; if the form is sesquilinear, $\langle x, y\rangle=x^{*} M y$ for all $x, y \in \mathbb{C}^{n}$, where the superscript $*$ denotes the conjugate transpose. The form is said to be nondegenerate when $M$ is nonsingular.

A bilinear form is symmetric if $\langle x, y\rangle=\langle y, x\rangle$ or, equivalently, if $M^{T}=M$, and skew-symmetric if $\langle x, y\rangle=-\langle y, x\rangle$ or, equivalently, if $M^{T}=-M$. A sesquilinear form is Hermitian if $\langle x, y\rangle=\overline{\langle y, x\rangle}$ and skew-Hermitian if $\langle x, y\rangle=-\overline{\langle y, x\rangle}$. The matrices associated with such forms are Hermitian and skew-Hermitian, respectively.

We will use the term scalar product to mean a nondegenerate bilinear or sesquilinear form on $\mathbb{K}^{n}$. When we have more than one scalar product under consideration, we will denote $\langle x, y\rangle$ by $\langle x, y\rangle_{\mathrm{M}}$, using the matrix $M$ defining the form as a subscript to distinguish the forms under discussion.

2.2. Adjoints. The adjoint of $A$ with respect to the scalar product $\langle\cdot, \cdot\rangle_{\mathrm{M}}$, denoted by $A^{\star}$, is uniquely defined by the property $\langle A x, y\rangle_{\mathrm{M}}=\left\langle x, A^{\star} y\right\rangle_{\mathrm{M}}$ for all $x$, $y \in \mathbb{K}^{n}$. It can be shown that the adjoint is given explicitly by

$$
A^{\star}= \begin{cases}M^{-1} A^{T} M & \text { for bilinear forms, } \\ M^{-1} A^{*} M & \text { for sesquilinear forms. }\end{cases}
$$

The following properties of adjoint, all analogous to properties of transpose (or conjugate transpose) follow easily, and hold for all scalar products.

Lemma 2.1. $(A+B)^{\star}=A^{\star}+B^{\star},(A B)^{\star}=B^{\star} A^{\star},\left(A^{-1}\right)^{\star}=\left(A^{\star}\right)^{-1}$ and

$$
(\alpha A)^{\star}= \begin{cases}\alpha A^{\star} & \text { for bilinear forms, } \\ \bar{\alpha} A^{\star} & \text { for sesquilinear forms. }\end{cases}
$$

The involutory property $\left(A^{\star}\right)^{\star}=A$ does not hold for all scalar products; this issue is discussed in section 2.4. Adjoints of rank-one matrices will often be needed,

$$
\left(y z^{T} M\right)^{\star}= \begin{cases}z y^{T} M & \text { for symmetric bilinear forms, } \\ -z y^{T} M & \text { for skew-symmetric bilinear forms. }\end{cases}
$$

Replacing ${ }^{T}$ by ${ }^{*}$ on both sides of (2.1) gives the corresponding results for Hermitian and skew-Hermitian sesquilinear forms respectively.

2.3. Lie and Jordan algebras. Associated with $\langle\cdot, \cdot\rangle_{\mathrm{M}}$ is a Lie algebra $\mathbb{L}$ and a Jordan algebra $\mathbb{J}$, defined by

$$
\begin{aligned}
\mathbb{L} & :=\left\{A \in \mathbb{K}^{n \times n}:\langle A x, y\rangle_{\mathrm{M}}=-\langle x, A y\rangle_{\mathrm{M}} \forall x, y \in \mathbb{K}^{n}\right\}=\left\{A \in \mathbb{K}^{n \times n}: A^{\star}=-A\right\}, \\
\mathbb{J} & :=\left\{A \in \mathbb{K}^{n \times n}:\langle A x, y\rangle_{\mathrm{M}}=\langle x, A y\rangle_{\mathrm{M}} \forall x, y \in \mathbb{K}^{n}\right\}=\left\{A \in \mathbb{K}^{n \times n}: A^{\star}=A\right\} .
\end{aligned}
$$

All the structured matrices considered in this paper belong to one of these two classes. Note that $\mathbb{L}$ and $\mathbb{J}$ are linear subspaces of $\mathbb{K}^{n \times n}$. Table 2.1 shows a sample of wellknown structured matrices in some $\mathbb{L}$ or $\mathbb{J}$ associated with a scalar product.

2.4. Orthosymmetric and unitary scalar products. Scalar products for which vector orthogonality is a symmetric relation, i.e.,

$$
\langle x, y\rangle_{\mathrm{M}}=0 \Leftrightarrow\langle y, x\rangle_{\mathrm{M}}=0, \forall x, y \in \mathbb{K}^{n},
$$


TABLE 2.1

Structured matrices associated with some orthosymmetric scalar products.

$R=\left[\begin{array}{l}. \\ 1\end{array}\right], J=\left[\begin{array}{cc}0 & I_{n} \\ -I_{n} & 0\end{array}\right], \Sigma_{p, q}=\left[\begin{array}{cc}I_{p} & 0 \\ 0 & -I_{q}\end{array}\right] \in \mathbb{R}^{n \times n}$ with $p+q=n$.

\begin{tabular}{|c|c|c|c|c|}
\hline Space & $\mathbf{M}$ & $\begin{array}{c}\text { Adjoint } \\
A^{\star}\end{array}$ & $\begin{array}{l}\text { Jordan Algebra } \\
\mathbb{J}=\left\{A: A^{\star}=A\right\}\end{array}$ & $\begin{array}{c}\text { Lie Algebra } \\
\mathbb{L}=\left\{A: A^{\star}=-A\right\}\end{array}$ \\
\hline \multicolumn{5}{|c|}{ Symmetric bilinear forms } \\
\hline $\mathbb{R}^{n}$ & $I$ & $A^{T}$ & Symmetrics & Skew-symmetrics \\
\hline $\mathbb{C}^{n}$ & $I$ & $A^{T}$ & Complex symmetrics & Cplx skew-symmetrics \\
\hline $\mathbb{R}^{n}$ & $\Sigma_{p, q}$ & $\Sigma_{p, q} A^{T} \Sigma_{p, q}$ & Pseudo symmetrics & Pseudo skew-symmetrics \\
\hline $\mathbb{C}^{n}$ & $\Sigma_{p, q}$ & $\Sigma_{p, q} A^{T} \Sigma_{p, q}$ & Cplx pseudo-symm. & Cplx pseudo-skew-symm. \\
\hline $\mathbb{R}^{n}$ & $R$ & $R A^{T} R$ & Persymmetrics & Perskew-symmetrics \\
\hline \multicolumn{5}{|c|}{ Skew-symmetric bilinear forms } \\
\hline $\mathbb{R}^{2 n}$ & $J$ & $-J A^{T} J$ & Skew-Hamiltonians & Hamiltonians \\
\hline $\mathbb{C}^{2 n}$ & $J$ & $-J A^{T} J$ & Cplx $J$-skew-symm. & Complex $J$-symmetrics \\
\hline
\end{tabular}

\begin{tabular}{|c|c|c|c|c|}
\hline \multicolumn{5}{|c|}{ Hermitian sesquilinear forms } \\
\hline $\mathbb{C}^{n}$ & $I$ & $A^{*}$ & Hermitian & Skew-Hermitian \\
\hline $\mathbb{C}^{n}$ & $\Sigma_{p, q}$ & $\Sigma_{p, q} A^{*} \Sigma_{p, q}$ & Pseudo Hermitian & Pseudo skew-Hermitian \\
\hline
\end{tabular}

\begin{tabular}{|l|l|l|l|l|}
\hline \multicolumn{5}{|c|}{ Skew-Hermitian sesquilinear forms } \\
\hline $\mathbb{C}^{2 n}$ & $J$ & $-J A^{*} J$ & $J$-skew-Hermitian & $J$-Hermitian \\
\hline
\end{tabular}

will be referred to as orthosymmetric scalar products $[13]$. One can show that $\langle\cdot, \cdot\rangle_{\mathrm{M}}$ is orthosymmetric if and only if it satisfies any one (and hence all) of the following equivalent properties:

1. $\mathbb{K}^{n \times n}=\mathbb{L} \oplus \mathbb{J}$.

2. The adjoint with respect to $\langle\cdot, \cdot\rangle_{\mathrm{M}}$ is involutory, i.e., $\left(A^{\star}\right)^{\star}=A, \forall A \in \mathbb{K}^{n \times n}$.

3. $M=\alpha M^{T}$ with $\alpha= \pm 1$ for bilinear forms; $M=\alpha M^{*}$ with $\alpha \in \mathbb{C},|\alpha|=1$ for sesquilinear forms.

See [13, Thm. A.4] for a proof of this equivalence along with a list of additional equivalent properties. The third property says that orthosymmetric bilinear forms are always either symmetric or skew-symmetric. On the other hand, an orthosymmetric sesquilinear form $\langle x, y\rangle_{\mathrm{M}}=x^{*} M y$, where $M=\alpha M^{*},|\alpha|=1, \alpha \in \mathbb{C}$ is always closely tied to a Hermitian form: defining the Hermitian matrix $H=\bar{\alpha}^{1 / 2} M$ gives $\langle x, y\rangle_{\mathrm{H}}=\bar{\alpha}^{1 / 2}\langle x, y\rangle_{\mathrm{M}}$, for all $x, y \in \mathbb{C}^{n}$. Consequently, the Jordan algebra of $\langle\cdot, \cdot\rangle_{\mathrm{H}}$ is identical to the Jordan algebra of $\langle\cdot, \cdot\rangle_{\mathrm{M}}$ :

$$
\langle A x, y\rangle_{\mathrm{H}}=\langle x, A y\rangle_{\mathrm{H}} \Leftrightarrow \bar{\alpha}^{1 / 2}\langle A x, y\rangle_{\mathrm{M}}=\bar{\alpha}^{1 / 2}\langle x, A y\rangle_{\mathrm{M}} \Leftrightarrow\langle A x, y\rangle_{\mathrm{M}}=\langle x, A y\rangle_{\mathrm{M}} .
$$

Similarly, the Lie algebra of $\langle\cdot, \cdot\rangle_{\mathrm{H}}$ and $\langle\cdot, \cdot\rangle_{\mathrm{M}}$ are also identical. Thus a result established for Hermitian sesquilinear forms immediately translates into a corresponding result for orthosymmetric sesquilinear forms. Up to a scalar multiple, then, there are really only three distinct types of orthosymmetric scalar products: symmetric and skew-symmetric bilinear, and Hermitian sesquilinear. We will, however, continue to include separately stated results (without separate proofs) for skew-Hermitian forms 
for convenience, as this is a commonly occurring special case.

The results in this paper hold only for orthosymmetric scalar products, which as we have just seen are those for which the useful and simplifying property $\left(A^{\star}\right)^{\star}=A$ holds for all matrices [13].

Some of our results will require the extra property that the scalar product $\langle\cdot, \cdot\rangle_{\mathrm{M}}$ is also unitary, that is, $\beta M$ is unitary for some $\beta>0$ [13]. One can show that in unitary scalar products, "the stars commute", i.e, $\left(A^{*}\right)^{\star}=\left(A^{\star}\right)^{*}$ for all $A \in \mathbb{K}^{n \times n}$ [13, Thm. A.7.]. Finally, note that important classes of structured matrices arise in the context of scalar products that are both orthosymmetric and unitary, as witnessed by the entries in Table 2.1 (all of which with $\alpha= \pm 1$ and $\beta=1$ ). The results in this paper are not confined to just the examples in the table, however.

2.5. Projections. Projections that map $x$ to the zero vector form a key part in our solution to the structured mapping problems.

Since the matrix $M$ of the scalar product is nonsingular, given a nonzero $x \in \mathbb{K}^{n}$ one can always construct many $w \in \mathbb{K}^{n}$ such that $\langle w, x\rangle_{\mathrm{M}}=1$. For example, when $x$ is nonisotropic (i.e $\langle x, x\rangle_{\mathrm{M}} \neq 0$ ), $w=x /\langle x, x\rangle_{\mathrm{M}}$ will work for bilinear forms, and $w=x /{\overline{\langle x, x\rangle_{\mathrm{M}}}}$ can be used for sesquilinear forms. If $x$ is isotropic (i.e $\langle x, x\rangle_{\mathrm{M}}=0$ ), choose $k$ so that $x_{k} \neq 0$; then $w=M^{-T} e_{k} / x_{k}$ will have the desired property for bilinear forms, and $w=M^{-*} e_{k} / \bar{x}_{k}$ will work for sesquilinear forms.

With $w$ chosen so that $\langle w, x\rangle_{\mathrm{M}}=1$, it is easy to show that for bilinear forms, $x w^{T} M$ is idempotent and hence a projection with range $\operatorname{span}\{x\}$. Replacing ${ }^{T}$ by * gives a similar result for sesquilinear forms. The complementary projections $P_{w}$ defined by

$$
P_{w}:=\left\{\begin{array}{lll}
I-x w^{T} M, & \langle w, x\rangle_{\mathrm{M}}=1 & \text { for bilinear forms, } \\
I-x w^{*} M, & \langle w, x\rangle_{\mathrm{M}}=1 & \text { for sesquilinear forms }
\end{array}\right.
$$

have kernel $\operatorname{span}\{x\}$, and in particular map $x$ to the zero vector. Expressions for the adjoint of $P_{w}$ will be useful in the calculations ahead:

$$
P_{w}^{\star}= \begin{cases}I-w x^{T} M & \text { for symmetric bilinear forms, } \\ I+w x^{T} M & \text { for skew-symmetric bilinear forms } \\ I-w x^{*} M & \text { for Hermitian sesquilinear forms, } \\ I+w x^{*} M & \text { for skew-Hermitian sesquilinear forms }\end{cases}
$$

3. The existence problem. Throughout the rest of the paper we assume that $x, b \in \mathbb{K}^{n}$ with $x \neq 0$, but that any $b$ is allowed unless otherwise stated. As a preliminary step towards solving the existence problem, we show that the projections given in (2.2) can be used to construct maps that send $x$ to $b$.

Lemma 3.1. Let $x \neq 0$, and let $w \in \mathbb{K}^{n}$ be chosen so that $\langle w, x\rangle_{\mathrm{M}}=1$. Then ${ }^{ \pm} B_{w} x=b$, where ${ }^{ \pm} B_{w} x$ is defined by

$$
{ }^{ \pm} B_{w}:= \begin{cases}b w^{T} M \pm\left(b w^{T} M\right)^{\star} P_{w} & \text { for bilinear forms } \\ b w^{*} M \pm\left(b w^{*} M\right)^{\star} P_{w} & \text { for sesquilinear forms. }\end{cases}
$$

Note that ${ }^{+} B_{w}$ and ${ }^{-} B_{w}$ have rank at most two.

Proof. Since $P_{w} x=0$ and $\langle w, x\rangle_{\mathrm{M}}=1$, we immediately conclude ${ }^{ \pm} B_{w} x=b$. Next, by $(2.1)$ we see that in the bilinear case, $\left(b w^{T} M\right)^{\star} P_{w}= \pm w b^{T} M P_{w}$ which is a rank one matrix, and hence ${ }^{+} B_{w},{ }^{-} B_{w}$ are the sum of two matrices of rank one. The proof in the sesquilinear case is similar. 
Thus, since ${ }^{+} B_{w},{ }^{-} B_{w}$ are always solutions to the unstructured mapping problem, they should be consistent with (1.1), which captures all solutions. Now $\langle w, x\rangle_{\mathrm{M}}=1$ implies $w^{T} M x=1$ in the bilinear case. Since any row vector $u^{T}$ with the property $u^{T} x=1$ is a generalized inverse $x^{\dagger}$ for the map $x: \mathbb{R} \rightarrow \mathbb{R}^{n}$, we can take $x^{\dagger}$ to be $w^{T} M$. Rewriting (3.1) for the bilinear case we get

$$
{ }^{ \pm} B_{w}=b x^{\dagger} \pm M^{-1} M^{T} w b^{T}\left(I-x x^{\dagger}\right) .
$$

Letting $Z= \pm M^{-1} M^{T} w b^{T}$, we see that (3.2) is indeed of the form given by Trenkler in (1.1). The argument for the sesquilinear case is similar, with the role of $x^{\dagger}$ being played by $w^{*} M$. It is worth observing that once the parameter $w^{T}$ is chosen, both $x^{\dagger}$ and $Z$ in (3.2) are determined, and thus we are confining our attention to a constrained subset of the maps given by (1.1).

We have still to determine when a structured solution exists, and the role ${ }^{+} B_{w}$, ${ }^{-} B_{w}$ play in such a solution. The next theorem characterizes pairs of vectors $x, b$ for which there exists $A \in \mathbb{L}$ or $\mathbb{J}$ such that $A x=b$. Observe that sometimes a condition on $\langle b, x\rangle_{\mathrm{M}}$ is needed, while in other cases a solution exists with no conditions at all. When a structured solution exists, we show that either ${ }^{-} B_{w}$ or ${ }^{+} B_{w}$ will be in the Lie or Jordan algebra, thus yielding a constructive proof of existence.

THEOREM 3.2 (Existence for $\mathbb{L}, \mathbb{J}$ ). Let $\langle\cdot, \cdot\rangle_{\mathrm{M}}$ be an orthosymmetric scalar product, and let $\mathbb{S}$ denote the corresponding Lie or Jordan algebra. Then for any given pair of vectors $x, b \in \mathbb{K}^{n}$ with $x \neq 0$, there exists $A \in \mathbb{S}$ such that $A x=b$ if and only if the conditions given in the following table hold:

\begin{tabular}{|c|c|c|}
\hline Bilinear Form & $\mathbb{J}$ & $\mathbb{L}$ \\
\hline symmetric & always & $\langle b, x\rangle_{\mathrm{M}}=0$ \\
\hline skew-symmetric & $\langle b, x\rangle_{\mathrm{M}}=0$ & always \\
\hline \hline Sesquilinear Form & $\mathbb{J}$ & $\mathbb{L}$ \\
\hline Hermitian & $\langle b, x\rangle_{\mathrm{M}} \in \mathbb{R}$ & $\langle b, x\rangle_{\mathrm{M}} \in i \mathbb{R}$ \\
\hline skew-Hermitian & $\langle b, x\rangle_{\mathrm{M}} \in i \mathbb{R}$ & $\langle b, x\rangle_{\mathrm{M}} \in \mathbb{R}$ \\
\hline
\end{tabular}

Proof. $(\Rightarrow)$ Since $A x=b$, in all cases we have

$$
\begin{aligned}
& A \in \mathbb{J} \Rightarrow\langle b, x\rangle_{\mathrm{M}}=\langle A x, x\rangle_{\mathrm{M}}=\langle x, A x\rangle_{\mathrm{M}}=\langle x, b\rangle_{\mathrm{M}} . \\
& A \in \mathbb{L} \Rightarrow\langle b, x\rangle_{\mathrm{M}}=\langle A x, x\rangle_{\mathrm{M}}=\langle x,-A x\rangle_{\mathrm{M}}=-\langle x, b\rangle_{\mathrm{M}} .
\end{aligned}
$$

For symmetric bilinear forms, $\langle b, x\rangle_{\mathrm{M}}=\langle x, b\rangle_{\mathrm{M}}$ for all $x, b \in \mathbb{K}^{n}$. Hence $A \in \mathbb{L}$ implies that $\langle b, x\rangle_{\mathrm{M}}=-\langle x, b\rangle_{\mathrm{M}}$ so that $\langle b, x\rangle_{\mathrm{M}}=0$.

For skew-symmetric bilinear forms, $\langle b, x\rangle_{\mathrm{M}}=-\langle x, b\rangle_{\mathrm{M}}$ for all $x, b \in \mathbb{K}^{n}$. Hence $A \in \mathbb{J}$ implies that $\langle b, x\rangle_{\mathrm{M}}=-\langle x, b\rangle_{\mathrm{M}}$ and therefore $\langle b, x\rangle_{\mathrm{M}}=0$.

For Hermitian sesquilinear forms, $\overline{\langle b, x\rangle_{\mathrm{M}}}=\langle x, b\rangle_{\mathrm{M}}$ for all $x, b \in \mathbb{C}^{n}$. Hence $A \in \mathbb{J}$ implies that $\langle b, x\rangle_{\mathrm{M}}=\langle x, b\rangle=\overline{\langle b, x\rangle_{\mathrm{M}}}$ so that $\langle b, x\rangle_{\mathrm{M}} \in \mathbb{R}$. Also, $A \in \mathbb{L}$ implies that $\langle b, x\rangle_{\mathrm{M}}=-\langle x, b\rangle_{\mathrm{M}}=-{\overline{\langle b, x\rangle_{\mathrm{M}}}}$ and therefore $\langle b, x\rangle_{\mathrm{M}} \in i \mathbb{R}$. The skew-Hermitian case follows from the Hermitian sesquilinear case.

$(\Leftarrow)$ For each of the eight cases we can give a concrete example of a matrix in $\mathbb{L}$ or $\mathbb{J}$ that maps $x$ to $b$. By Lemma 3.1 we know that ${ }^{+} B_{w},{ }^{-} B_{w}$ as defined in (3.1) map $x$ to $b$. It suffices to prove that when the conditions stated in the table hold, ${ }^{+} B_{w} \in \mathbb{J}$, and ${ }^{-} B_{w} \in \mathbb{L}$. We first consider the symmetric bilinear case. Using Lemma 2.1, the 
expressions for the adjoints given in (2.1) and (2.3), and the symmetry of the scalar product $\left(M=M^{T},\langle u, v\rangle_{\mathrm{M}}=\langle v, u\rangle_{\mathrm{M}}\right.$ for all $\left.u, v\right)$, we have

$$
\begin{aligned}
{ }^{+} B_{w}^{\star} & =\left(b w^{T} M\right)^{\star}+P_{w}^{\star}\left(b w^{T} M\right) \\
& =\left(b w^{T} M\right)^{\star}+\left(I-w x^{T} M\right) b w^{T} M \\
& =\left(b w^{T} M\right)^{\star}-w\left(x^{T} M b\right) w^{T} M+b w^{T} M \\
& =\left(b w^{T} M\right)^{\star}-\left(w b^{T} M\right)\left(x w^{T} M\right)+b w^{T} M \\
& =\left(b w^{T} M\right)^{\star}-\left(b w^{T} M\right)^{\star}\left(x w^{T} M\right)+b w^{T} M \\
& =\left(b w^{T} M\right)^{\star}\left(I-x w^{T} M\right)+b w^{T} M={ }^{+} B_{w}
\end{aligned}
$$

so that ${ }^{+} B_{w} \in \mathbb{J}$ for all $x, b \in \mathbb{K}^{n}$ with $x \neq 0$. To see why ${ }^{-} B_{w} \in \mathbb{L}$, we need to expand ${ }^{-} B_{w}$ :

$$
\begin{aligned}
{ }^{-} B_{w} & =\left(b w^{T} M\right)-\left(b w^{T} M\right)^{\star} P_{w} \\
& =\left(b w^{T} M\right)-\left(b w^{T} M\right)^{\star}+\left(b w^{T} M\right)^{\star} x w^{T} M \\
& =\left(b w^{T} M\right)-\left(b w^{T} M\right)^{\star}+\langle b, x\rangle_{\mathrm{M}} w w^{T} M \\
& =\left(b w^{T} M\right)-\left(b w^{T} M\right)^{\star}
\end{aligned}
$$

since $\langle b, x\rangle_{\mathrm{M}}=0$ by hypothesis. Now $\left(b w^{T} M\right)-\left(b w^{T} M\right)^{\star} \in \mathbb{L}$, since the adjoint is involutory for orthosymmetric scalar products. Thus ${ }^{-} B_{w} \in \mathbb{L}$.

The proof for the skew-symmetric bilinear case is similar. In the Hermitian sesquilinear case, we have

$$
\begin{aligned}
{ }^{+} B_{w}^{\star} & =\left(b w^{*} M\right)^{\star}+P_{w}^{\star}\left(b w^{*} M\right) \\
& =\left(b w^{*} M\right)^{\star}+b w^{*} M-\langle x, b\rangle_{\mathrm{M}} w w^{*} M .
\end{aligned}
$$

On the other hand,

$$
\begin{aligned}
{ }^{+} B_{w} & =b w^{*} M+\left(b w^{*} M\right)^{\star} P_{w} \\
& =b w^{*} M+\left(b w^{*} M\right)^{\star}-\langle b, x\rangle_{\mathrm{M}} w w^{*} M .
\end{aligned}
$$

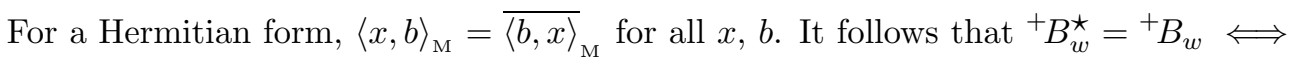
$\langle x, b\rangle_{\mathrm{M}}=\langle b, x\rangle_{\mathrm{M}} \Longleftrightarrow\langle b, x\rangle_{\mathrm{M}} \in \mathbb{R}$. The proof for skew-Hermitian sesquilinear forms follows with minor adaptations.

Theorem 3.2 unifies and generalizes existence results in [9] for real skew-symmetric matrices, in [8] and [16] for symmetric and Hermitian matrices, in [18] for complex symmetric and skew-Hermitian structures, and in [15, Lem. 5.1] for real persymmetric matrices, which are particular instances of Lie and Jordan algebras associated with different bilinear and sesquilinear forms on $\mathbb{R}^{n}$ and $\mathbb{C}^{n}$ (see Table 2.1).

4. The characterization problem. We turn now to the task of determining the set of all matrices that map $x$ to $b$ and belong to a Lie or Jordan algebra.

LEMMA 4.1. Let $\mathbb{S}$ denote the Lie or Jordan algebra of any orthosymmetric scalar product. Then

(a) $A \in \mathbb{S} \Longrightarrow Q^{\star} A Q \in \mathbb{S}$ for all $Q$; that is, $\star$-congruence preserves $\mathbb{L}$ and $\mathbb{J}$ structures.

(b) $\left\{P_{w}^{\star} S P_{w}: S \in \mathbb{S}\right\} \subseteq\{A \in \mathbb{S}: A x=0\}$, where $P_{w}$ is any particular one of the projection matrices defined in $(2.2)$.

(c) For any $w \in \mathbb{K}^{n}$ such that $\langle w, x\rangle_{M}=1, A \in \mathbb{S}, A x=0 \Longrightarrow A=P_{w}^{\star} A P_{w}$. 
Proof. (a)This is a direct consequence of adjoint being involutory in orthosymmetric scalar products.

(b) Follows immediately from the fact that $P_{w}=0$, together with (a).

(c) For any bilinear form, $A \in \mathbb{S} \Longrightarrow A= \pm A^{\star}= \pm M^{-1} A^{T} M \Longrightarrow M A=$ $\pm A^{T} M \Longrightarrow x^{T} M A= \pm x^{T} A^{T} M$. But $A x=0$. Hence $x^{T} M A=0$. From (2.2), we have $P_{w}=I-x w^{T} M$. Hence $A P_{w}=A-A x w^{T} M=A$, since $A x=0$. Combining this with the expression for $P_{w}^{\star}$ from $(2.3)$, we obtain

$$
P_{w}^{\star} A P_{w}=P_{w}^{\star} A=\left(I \pm w x^{T} M\right) A=A
$$

since $x^{T} M A=0$. The proof for sesquilinear forms follows along the same lines. $\square$ The complete solution to the homogeneous mapping problem can now be described.

TheOREM 4.2 (Characterization for $\mathbb{J}$ and $\mathbb{L}$ : homogeneous case). Let $\mathbb{S}$ denote the Lie or Jordan algebra of any orthosymmetric scalar product space. Given $x \in \mathbb{K}^{n}$ with $x \neq 0$, and $w \in \mathbb{K}^{n}$ such that $\langle w, x\rangle_{\mathrm{M}}=1$,

$$
\{A \in \mathbb{S}: A x=0\}=\left\{P_{w}^{\star} S P_{w}: S \in \mathbb{S}\right\}
$$

where $P_{w}$ is defined in (2.2).

Proof. Follows immediately by combining (b) and (c) of Lemma 4.1.

Corollary 4.3. If $v, w \in \mathbb{K}^{n}$, with $\langle v, x\rangle_{\mathrm{M}}=\langle w, x\rangle_{\mathrm{M}}=1$, then

$$
\left\{P_{v}^{\star} S P_{v}: S \in \mathbb{S}\right\}=\left\{P_{w}^{\star} S P_{w}: S \in \mathbb{S}\right\}
$$

Thus we have several representations of the set of solutions to the homogeneous mapping problem. Now if $A, B \in \mathbb{S}$ are such that $A x=B x=b$, then $(A-B) x=0$. By Proposition 4.2, $A-B=P_{w}^{\star} S P_{w}$, or equivalently, $A=B+P_{w}^{\star} S P_{w}$ for some $S \in \mathbb{S}$. This relationship between the solution sets of the homogeneous system $A x=0$ and the non-homogeneous system $A x=b$, when $x$ is unknown and $A, b$ are fixed is the same as when the roles of $A$ and $x$ are interchanged as in the mapping problem:

$$
\{A \in \mathbb{S}: A x=b\}=B+\{A \in \mathbb{S}: A x=0\},
$$

where $B$ is any particular solution of the non-homogeneous mapping problem. We can now solve the characterization part of the mapping problem for $\mathbb{L}$ and $\mathbb{J}$.

THEOREM 4.4 (Characterization for $\mathbb{J}$ and $\mathbb{L}$ : non-homogeneous case). Let $\mathbb{J}$ and $\mathbb{L}$ be the Jordan and Lie algebras of any orthosymmetric scalar product on $\mathbb{K}^{n}$. Let $x, b \in \mathbb{K}^{n}$ and $x \neq 0$. Choose any $v, w \in \mathbb{K}^{n}$ such that $\langle v, x\rangle_{\mathrm{M}}=\langle w, x\rangle_{\mathrm{M}}=1$, and use $v$ and $w$ to define $P_{v},{ }^{ \pm} B_{w}$ as in (2.2) and (3.1) respectively. Consider the following sets:

$$
\begin{array}{ll}
\mathcal{J}=\{A \in \mathbb{J}: A x=b\}, & \mathcal{J}_{+}=\left\{{ }^{+} B_{w}+P_{v}^{\star} S P_{v}: S \in \mathbb{J}\right\} \\
\mathcal{L}=\{A \in \mathbb{L}: A x=b\}, & \mathcal{L}_{-}=\left\{{ }^{-} B_{w}+P_{v}^{\star} L P_{v}: L \in \mathbb{L}\right\}
\end{array}
$$

Then $\mathcal{J}$ is either empty or $\mathcal{J}_{+}$, and $\mathcal{L}$ is either empty or $\mathcal{L}_{-}$, depending on the conditions described in the following table: 


\begin{tabular}{|c|c|c|}
\hline Bilinear Form & $\mathcal{J}$ & $\mathcal{L}$ \\
\hline symmetric & $\mathcal{J}_{+}$always & $\begin{array}{c}\mathcal{L}_{-} \text {if }\langle b, x\rangle_{\mathrm{M}}=0 \\
\emptyset, \text { otherwise }\end{array}$ \\
\hline skew-symmetric & $\begin{array}{c}\mathcal{J}_{+} \text {if }\langle b, x\rangle_{\mathrm{M}}=0 \\
\emptyset, \text { otherwise }\end{array}$ & $\mathcal{L}_{-}$always \\
\hline Sesquilinear Form & $\mathcal{J}$ & $\mathcal{L}$ \\
\hline Hermitian & $\begin{array}{c}\mathcal{J}_{+} \text {if }\langle b, x\rangle_{\mathrm{M}} \in \mathbb{R} \\
\emptyset, \text { otherwise }\end{array}$ & $\begin{array}{c}\mathcal{L}_{-} \text {if }\langle b, x\rangle_{\mathrm{M}} \in i \mathbb{R} \\
\emptyset, \text { otherwise }\end{array}$ \\
\hline skew-Hermitian & $\begin{array}{c}\mathcal{J}_{+} \text {if }\langle b, x\rangle_{\mathrm{M}} \in i \mathbb{R} \\
\emptyset, \text { otherwise }\end{array}$ & $\begin{array}{c}\mathcal{L}_{-} \text {if }\langle b, x\rangle_{\mathrm{M}} \in \mathbb{R} \\
\emptyset, \text { otherwise }\end{array}$ \\
\hline
\end{tabular}

Proof. Theorem 3.2 gives conditions under which a solution to the mapping problem exists, and shows that ${ }^{+} B_{w}$ and ${ }^{-} B_{w}$ are particular solutions when these conditions are satisfied. Combining these results with the observation that $A-B$ is a solution to the homogeneous problem whenever $A$ and $B$ are particular solutions gives the desired result via Theorem 4.2 .

A more general problem for Hermitian, and real symmetric matrices in particular, was considered by Sun [16, Lem. 1.4]. For given matrices $X, B \in \mathbb{K}^{n \times \ell}$, Sun gave a characterization of the set

$$
\mathcal{H}=\left\{A \in \mathbb{K}^{n \times n}: A^{*}=A \text { and } A X=B\right\}
$$

in terms of the pseudo-inverse $X^{+}$of $X$, and the complementary orthogonal projections $\Pi_{X}=X X^{+}$, and $\Pi_{X^{\perp}}=I-\Pi_{X}$. He proved that $\mathcal{H} \neq \emptyset$ if and only if two conditions are satisfied: $B \Pi_{X^{*}}=B$ and $\Pi_{X} B X^{+}$is Hermitian. In this case $\mathcal{H}$ can be expressed as

$$
\mathcal{H}=\left\{B X^{+}+\left(B X^{+}\right)^{*} \Pi_{X^{\perp}}+\Pi_{X^{\perp}} S \Pi_{X^{\perp}}: \quad S^{*}=S, S \in \mathbb{K}^{n \times n}\right\} .
$$

When $\ell=1$, writing $X, B$ as $x, b$ respectively, we get $\Pi_{x}=x x^{*} /\left(x^{*} x\right)$, and $x^{+}=$ $x^{*} /\left(x^{*} x\right)$. Since $\Pi_{x^{*}}=1$, the conditions for $\mathcal{H}$ to be nonempty reduce to requiring that $\Pi_{x} b x^{+}$be Hermitian. A simple calculation shows that this happens if and only if $x^{*} b$ is real, which is in agreement with the condition in Theorem 4.4. Sun's characterization of $\mathcal{H}$ becomes

$$
\mathcal{H}=\left\{\frac{b x^{*}}{x^{*} x}+\frac{x^{*} b}{x^{*} x} \Pi_{x}+\Pi_{x} S \Pi_{x}, S^{*}=S\right\},
$$

which corresponds to $\mathcal{J}_{+}$in Theorem 4.4 with $M=I$ and the special choice $v=$ $w=x /\left(x^{*} x\right)$. This choice of $w$ corresponds to using an orthogonal projection in the representation for $\mathcal{J}_{+}$, since $P_{v}$ is now $I-x x^{*} /\left(x^{*} x\right)$. Thus Sun's characterization is one among many given by Theorem 4.4 .

A similar analysis of the real symmetric case shows that the results of Theorem 3.2 and Theorem 4.4 are compatible with Sun's solution for the case $\ell=1$, and due to the freedom in the choice of $v$ and $w$, gives a more flexible description of the set of real symmetric matrices mapping $x$ to $b$.

5. Structured mappings with extremal properties. In Theorem 4.4 we introduced the notation

$$
\mathcal{J}=\{A \in \mathbb{J}: A x=b\}, \quad \mathcal{L}=\{A \in \mathbb{L}: A x=b\},
$$


for the sets of all structured solutions to the mapping problem. We now show how to find matrices in $\mathcal{J}$ or $\mathcal{L}$ with the extremal properties of minimal rank, minimal Frobenius norm, or minimal 2-norm and investigate their uniqueness.

5.1. Structured solutions of minimal rank. In what follows, we assume $b \neq 0$.

THEOREM 5.1 (Rank-one structured mappings). Let $\langle\cdot, \cdot\rangle_{\mathrm{M}}$ be an orthosymmetric scalar product, and let $\mathcal{S}$ denote either $\mathcal{J}$ or $\mathcal{L}$ as in (5.1). Assume $b \neq 0$. A necessary condition for the existence of a rank-one matrix in $\mathcal{S}$ is $\langle b, x\rangle_{\mathrm{M}} \neq 0$. Whenever this rank-one matrix exists, it is unique and given by

$$
A= \begin{cases}b b^{T} M /\langle b, x\rangle_{\mathrm{M}} & \text { for bilinear forms } \\ b b^{*} M /\langle b, x\rangle_{\mathrm{M}} & \text { for sesquilinear forms }\end{cases}
$$

Proof. Consider any rank-one matrix $A=u v^{T}$ such that $A x=b$ with $b \neq 0$. Since $b \in \operatorname{range}(A), u$ is a multiple of $b$, so without loss of generality we can take $u=b$.

Now suppose the orthosymmetric scalar product is bilinear, so $M^{T}= \pm M$. Since $M$ is nonsingular, there exists $z \in \mathbb{K}^{n}$ such that $v^{T}=z^{T} M$, and so $A=u v^{T}=b z^{T} M$. For $A \in \mathbb{L}$ or $\mathbb{J}$ we have $A^{\star}=\epsilon A$ with $\epsilon= \pm 1$, so by (2.1) we have $\pm z b^{T} M=\epsilon b z^{T} M$ and so $z b^{T}= \pm b z^{T}$. Thus $z=\alpha b$ and $A=\alpha b b^{T} M$. But $A x=b \Rightarrow \alpha b\left(b^{T} M x\right)=$ $b \Rightarrow \alpha\langle b, x\rangle_{\mathrm{M}}=1$, thus forcing $\langle b, x\rangle_{\mathrm{M}}$ to be nonzero, and uniquely determining $A$ by $A=b b^{T} M /\langle b, x\rangle_{\mathrm{M}}$. Similar reasoning applies for the sesquilinear case, leading to the formula $A=b b^{*} M /\langle b, x\rangle_{\mathrm{M}}$. $\square$

COROllary 5.2. Let $b \neq 0$. If $\langle b, x\rangle_{\mathrm{M}} \neq 0$, then either $\mathcal{S}$ is empty or there is $a$ unique $A \in \mathcal{S}$ with $\operatorname{rank}(A)=1$.

Proof. Follows immediately from Theorem 5.1 together with Theorem 4.4. $\square$

The particular cases of Corollary 5.2 are summarized in the following table. Substituting the choice of $w$ indicated in Table 5.1 into the formulas for ${ }^{+} B_{w},{ }^{-} B_{w}$ given in (3.1) yields the unique rank one mapping specified in Theorem 5.1. The extra condition in the Hermitian sesquilinear case comes from the result in Theorem 4.4. For brevity the skew-Hermitian sesquilinear case has been omitted.

TABLE 5.1

Rank-one structured mappings when $\langle b, x\rangle_{\mathrm{M}} \neq 0, \alpha=1 /\langle b, x\rangle_{\mathrm{M}}$

\begin{tabular}{|c|c|c|}
\hline Scalar Product & $\mathcal{J}$ & $\mathcal{L}$ \\
\hline symmetric bilinear & ${ }^{+} B_{w}$ with $w=\alpha b$ & $\mathcal{L}$ is empty \\
\hline skew-symmetric bilinear & $\mathcal{J}$ is empty & ${ }^{-} B_{w}$ with $w=\alpha b$ \\
\hline Hermitian sesquilinear & $\begin{array}{c}\text { If }\langle b, x\rangle_{\mathrm{M}} \in \mathbb{R} \backslash\{0\}, \\
+B_{w} \text { with } w=\alpha b \\
\text { Otherwise no mapping }\end{array}$ & $\begin{array}{c}\text { If }\langle b, x\rangle_{\mathrm{M}} \in i \mathbb{R} \backslash\{0\}, \\
-B_{w} \text { with } w=\bar{\alpha} b \\
\text { Otherwise no mapping }\end{array}$ \\
\hline
\end{tabular}

For nonzero $b$ we have seen that the condition $\langle b, x\rangle_{\mathrm{M}} \neq 0$, while necessary for the existence of structured rank-one mappings, is precisely the condition that precludes the existence of any structured mappings in certain cases (see Theorem 4.4). On the other hand, the table in Theorem 4.4 also shows that structured solution sets $\mathcal{S}$ are never empty when the condition $\langle b, x\rangle_{\mathrm{M}}=0$ is met. We turn to the question of determining what the minimal achievable rank is in this case. 
TheOREM 5.3 (Rank-two structured mappings). Let $\langle\cdot, \cdot\rangle_{\mathrm{M}}$ be an orthosymmetric scalar product, and let $\mathcal{S}$ denote either $\mathcal{J}$ or $\mathcal{L}$ as in (5.1). Consider any nonzero $x$, $b \in \mathbb{K}^{n}$. If $\langle b, x\rangle_{\mathrm{M}}=0$ then

$$
\min _{A \in \mathcal{S}} \operatorname{rank}(A)=2
$$

There are always infinitely many matrices in $\mathcal{S}$ attaining this minimal rank. Among these are ${ }^{-} B_{w} \in \mathcal{L}$ and ${ }^{+} B_{w} \in \mathcal{J}$, where ${ }^{-} B_{w},{ }^{+} B_{w}$ are given by (3.1), with any choice of $w \in \mathbb{K}^{n}$ such that $\langle w, x\rangle_{\mathrm{M}}=1$.

Proof. If $\langle b, x\rangle_{\mathrm{M}}=0$, then by Theorem 5.1, the minimum possible rank for matrices in $\mathcal{S}$ is 2 . We know ${ }^{+} B_{w},{ }^{-} B_{w}$ map $x$ to $b$ for all $w \in \mathbb{K}^{n}$ such that $\langle w, x\rangle_{\mathrm{M}}=1$, and from Theorem 3.2 it follows that ${ }^{+} B_{w} \in \mathbb{J}$ and ${ }^{-} B_{w} \in \mathbb{L}$ for all such $w$. Since ${ }^{+} B_{w}$, ${ }^{-} B_{w}$ are at most rank two, and since they cannot be rank-one, they are solutions of rank two.

5.2. Structured mappings of minimal Frobenius norm. Another important special property is minimal norm, since this is directly related to structured backward errors for linear systems and eigenvalue problems [18], [19] as well as the derivation of quasi-Newton methods [3]. We first consider the minimal Frobenius norm case, the minimal 2-norm case being treated in the next section. For symmetric or Hermitian structures, it is well-known [2], [3] that minimal Frobenius norm is achieved by

$$
A_{\mathrm{opt}}=\frac{b x^{*}+x b^{*}}{x^{*} x}-\frac{\left(b^{*} x\right)}{\left(x^{*} x\right)^{2}} x x^{*}
$$

We show how to generalize this result to all Lie and Jordan algebras associated with scalar products that are both orthosymmetric and unitary. We will need the next two lemmas to prove that, whenever it exists, the structured mapping of minimal Frobenius norm is unique.

LEMMA 5.4. In any real or complex inner product space, the associated norm $\|\cdot\|$ is strictly convex on independent vectors, that is,

$$
\|t u+(1-t) v\|<t\|u\|+(1-t)\|v\|, \quad 0<t<1
$$

for any linearly independent $u$ and $v$.

Proof. The Cauchy-Schwarz inequality implies that $\langle u, v\rangle+\langle v, u\rangle<2\|u\|\|v\|$ for linearly independent $u, v$. A straightforward calculation then establishes the result. $\square$

Lemma 5.5. For $b \neq 0$, the Frobenius norm is strictly convex on $\mathcal{S}(\mathcal{S}=\mathcal{J}, \mathcal{L})$.

Proof. Assuming $b \neq 0$, distinct $A, B \in \mathcal{S}$ are linearly independent. Since the Frobenius norm arises from the inner product $\langle A, B\rangle=\operatorname{tr}\left(A^{*} B\right)$, the result is immediate from Lemma 5.4.

Theorem 5.6 (Minimal Frobenius norm structured mapping). Let $\langle\cdot, \cdot\rangle_{\mathrm{M}}$ be a scalar product that is both orthosymmetric and unitary. Let $\mathcal{S}$ denote either $\mathcal{J}$ or $\mathcal{L}$ as in (5.1). If $\mathcal{S} \neq \emptyset$, the problem

$$
\min _{A \in \mathcal{S}}\|A\|_{F}
$$

has a unique solution given by

$$
A_{\mathrm{opt}}=\frac{b x^{*}}{x^{*} x}+\epsilon\left(\frac{b x^{*}}{x^{*} x}\right)^{\star}\left(I-\frac{x x^{*}}{x^{*} x}\right), \quad \epsilon= \begin{cases}1 & \text { if } \mathcal{S}=\mathcal{J}, \\ -1 & \text { if } \mathcal{S}=\mathcal{L}\end{cases}
$$


Moreover,

$$
\left\|A_{\mathrm{opt}}\right\|_{F}^{2}=2 \frac{\|b\|_{2}^{2}}{\|x\|_{2}^{2}}-\beta^{2} \frac{\left|\langle b, x\rangle_{\mathrm{M}}\right|^{2}}{\|x\|_{2}^{4}},
$$

where $\beta>0$ is such that $\beta M$ is unitary.

Proof. Let

$$
w= \begin{cases}M^{-T} \bar{x} /\left(x^{*} x\right) & \text { (bilinear forms) } \\ M^{-*} x /\left(x^{*} x\right) & (\text { sesquilinear forms })\end{cases}
$$

satisfying $\langle w, x\rangle_{\mathrm{M}}=1$. With this particular choice of $w, P_{w}$ in (2.2) and ${ }^{ \pm} B_{w}$ in (3.1) become

$$
P_{w}=I-\frac{x x^{*}}{x^{*} x}, \quad{ }^{ \pm} B_{w}=\frac{b x^{*}}{x^{*} x} \pm\left(\frac{b x^{*}}{x^{*} x}\right)^{\star} P_{w} .
$$

We know that ${ }^{+} B_{w}$ and ${ }^{-} B_{w}$ map $x$ to $b$ and that if $x, b$ satisfy the conditions in Theorem 3.2 then $\mathcal{S} \neq \emptyset$ and ${ }^{+} B_{w} \in \mathbb{J},{ }^{-} B_{w} \in \mathbb{L}$. Hence $A_{\mathrm{opt}}={ }^{ \pm} B_{w} \in \mathcal{S}$.

Let $\left\{x /\|x\|_{2}, u_{2}, \ldots, u_{n}\right\}$ be an orthonormal basis with respect to the standard inner product on $\mathbb{K}^{n}$. To show that $A_{\text {opt }}$ has minimal Frobenius norm we first observe that

$$
\|A x\|_{F}=\|b\|_{F}=\left\|A_{\mathrm{opt}} x\right\|_{F} \quad \forall A \in \mathcal{S} .
$$

The Characterization Theorem 4.4 with $v=w$ tells us that any $A \in \mathcal{S}$ can be written as $A=A_{\mathrm{opt}}+P_{w}^{\star} S P_{w}$ for some $S \in \mathbb{S}$. Premultiplying $u_{i}$ by $A$ and taking norm yields

(5.5) $\left\|A u_{i}\right\|_{2}^{2}=\left\|A_{\mathrm{opt}} u_{i}\right\|_{2}^{2}+\left\|P_{w}^{\star} S P_{w} u_{i}\right\|_{2}^{2}+2 \operatorname{Re}\left(\left(A_{\mathrm{opt}} u_{i}\right)^{*} P_{w}^{\star} S P_{w} u_{i}\right), \quad 2 \leq i \leq n$.

When $\langle\cdot, \cdot\rangle_{\mathrm{M}}$ is unitary, we claim that the last term on the right hand side of (5.5) always vanishes. To see that, we first consider the bilinear form case. Since the stars commute in a unitary scalar product and $x^{*} u_{i}=0, i=1: n-1$, we have

$$
\left(A_{\mathrm{opt}} u_{i}\right)^{*}= \pm u_{i}^{*}\left(\left(\frac{b x^{*}}{x^{*} x}\right)^{\star}\right)^{*}= \pm u_{i}^{*}\left(\frac{x b^{*}}{x^{*} x}\right)^{\star}= \pm\left(\frac{u_{i}^{*} M^{-1} \bar{b}}{x^{*} x}\right) x^{T} M=: \alpha x^{T} M
$$

and,

$$
\left(A_{\mathrm{opt}} u_{i}\right)^{*} P_{w}^{\star} S P_{w} u_{i}=\alpha x^{T} M\left(M^{-1}\left(I-\frac{\bar{x} x^{T}}{x^{*} x}\right) M\right) S u_{i}=\alpha\left(x^{T}-x^{T}\right) M S u_{i}=0 .
$$

Similarly, for sesquilinear forms, $\left(A_{\mathrm{opt}} u_{i}\right)^{*}=\alpha x^{*} M$ with $\alpha= \pm\left(u_{i}^{*} M^{-1} b\right) /\left(x^{*} x\right)$ and

$$
\left(A_{\mathrm{opt}} u_{i}\right)^{*} P_{w}^{\star} S P_{w} u_{i}=\alpha x^{*} M\left(M^{-1}\left(I-\frac{x x^{*}}{x^{*} x}\right) M\right) S u_{i}=\alpha\left(x^{*}-x^{*}\right) M S u_{i}=0 .
$$

Therefore from (5.5), $\left\|A u_{i}\right\|_{2} \geq\left\|A_{\mathrm{opt}} u_{i}\right\|_{2}, 2 \leq i \leq n$, and since $\left\{x /\|x\|_{2}, u_{2}, \ldots, u_{n}\right\}$ forms an orthonormal set on $\mathbb{K}^{n}$, one has

$$
\|A\|_{F}=\frac{\|A x\|_{2}}{\|x\|_{2}}+\sum_{i=2}^{n}\left\|A u_{i}\right\|_{2} \geq \frac{\left\|A_{\mathrm{opt}} x\right\|_{2}}{\|x\|_{2}}+\sum_{i=2}^{n}\left\|A_{\mathrm{opt}} u_{i}\right\|_{2}=\left\|A_{\mathrm{opt}}\right\|_{F}, \quad \forall A \in \mathcal{S},
$$


showing that $A_{\mathrm{opt}}$ has minimal Frobenius norm.

It is well-known that strictly convex functions have at most one minimizer [1, p.4]. Therefore Lemma 5.5 implies that $A_{\mathrm{opt}}$ is unique for $b \neq 0$. When $b=0, A_{\mathrm{opt}}=0$ is clearly unique.

Finally, for the Frobenius norm of $A_{\text {opt }}$ we have

$$
\left\|A_{\mathrm{opt}}\right\|_{F}^{2}=\left\|\frac{b x^{*}}{x^{*} x}\right\|_{F}^{2}+\left\|\left(\frac{b x^{*}}{x^{*} x}\right)^{\star} P_{w}\right\|_{F}^{2}+2 \epsilon \operatorname{Re}\left(\operatorname{tr}\left[\frac{x b^{*}}{x^{*} x}\left(\frac{b x^{*}}{x^{*} x}\right)^{\star} P_{w}\right]\right) .
$$

Note that

$$
\operatorname{tr}\left[\frac{x b^{*}}{x^{*} x}\left(\frac{b x^{*}}{x^{*} x}\right)^{\star} P_{w}\right]=\operatorname{tr}\left[\left(I-\frac{x x^{*}}{x^{*} x}\right) \frac{x b^{*}}{x^{*} x}\left(\frac{b x^{*}}{x^{*} x}\right)^{\star}\right]=\operatorname{tr}(0)=0
$$

and that $\left\|\left(b x^{*}\right) /\left(x^{*} x\right)\right\|_{F}^{2}=\|b\|_{2}^{2} /\|x\|_{2}^{2}$. Now using Lemma 2.1 and the fact that the stars commute in a unitary scalar product, we obtain

$$
\left\|\left(\frac{b x^{*}}{x^{*} x}\right)^{\star} P_{w}\right\|_{F}^{2}=\frac{\|b\|_{2}^{2}}{\|x\|_{2}^{2}}-\frac{\langle b, x\rangle_{\mathrm{M}}}{\left(x^{*} x\right)^{2}} \operatorname{tr}\left(u x^{*}\right),
$$

where

$$
u= \begin{cases}M^{-1} \bar{b} & \text { for bilinear forms } \\ M^{-1} b & \text { for sesquilinear forms }\end{cases}
$$

$\langle\cdot, \cdot\rangle_{M}$ being a unitary scalar product means that $\beta M$ is unitary for some $\beta>0$. Hence, $M^{-1}=\beta^{2} M^{*}$ and therefore,

$$
\operatorname{tr}\left(u x^{*}\right)=x^{*} u=\overline{u^{*} x}=\left\{\begin{array}{ll}
\beta^{2} \overline{b^{T} M x} & \text { (bilinear forms) } \\
\beta^{2} \overline{b^{*} M x} & \text { (sesquilinear forms) }
\end{array}\right\}=\beta^{2} \overline{\langle b, x\rangle}{ }_{\mathrm{M}}
$$

so that

$$
\left\|\left(\frac{b x^{*}}{x^{*} x}\right)^{\star} P_{w}\right\|_{F}^{2}=\frac{\|b\|_{2}^{2}}{\|x\|_{2}^{2}}-\beta^{2} \frac{\left|\langle b, x\rangle_{M}\right|^{2}}{\|x\|_{2}^{4}}
$$

which completes the proof. $\square$

5.3. Structured mappings of minimal 2-norm. From $A x=b$ it is clear that $\|b\|_{2} /\|x\|_{2}$ is always a lower bound for $\|A\|_{2}$. For a large class of scalar products Theorem 5.6 also yields an upper bound:

$$
\frac{\|b\|_{2}}{\|x\|_{2}} \leq \min _{A \in \mathcal{S}}\|A\|_{2} \leq \min _{A \in \mathcal{S}}\|A\|_{F} \leq \sqrt{2} \frac{\|b\|_{2}}{\|x\|_{2}},
$$

where $\mathcal{S}$ denotes either $\mathcal{J}$ or $\mathcal{L}$ as in (5.1). In this section we show that the lower bound is actually attained in any Lie or Jordan algebra of a scalar product that is both orthosymmetric and unitary.

Unlike the structured mapping of minimal Frobenius norm, mappings of minimal 2-norm in $\mathcal{S}$ are almost never unique. For example, consider the Jordan algebra of $n \times n$ symmetric matrices with $n \geq 3$, and take $x=e_{1}$ and $b=e_{2}$ to be the first 
and second column of the identity matrix, respectively. Then all matrices of the form $A=\operatorname{diag}\left(\left[\begin{array}{ll}0 & 1 \\ 1 & 0\end{array}\right], S\right)$ with $S$ symmetric and $\|S\|_{2} \leq 1$ satisfy $A^{T}=A, A x=b$, and have $\|A\|_{2}=\|b\|_{2} /\|x\|_{2}=1$. Indeed, this formula captures all symmetric matrices mapping $e_{1}$ to $e_{2}$ that have minimal 2-norm. We give similar characterizations of the sets of minimal 2-norm structured mappings for large classes of Lie and Jordan algebras.

5.3.1. Symmetric, skew-symmetric, and Hermitian mappings. We begin by solving the minimal 2-norm structured mapping problem for the five key structures

$$
\begin{aligned}
\operatorname{Sym}(n, \mathbb{K}) & =\left\{A \in \mathbb{K}^{n \times n}: A^{T}=A\right\}, \\
\operatorname{Skew}(n, \mathbb{K}) & =\left\{A \in \mathbb{K}^{n \times n}: A^{T}=-A\right\}, \\
\operatorname{Herm}(n, \mathbb{C}) & =\left\{A \in \mathbb{C}^{n \times n}: A^{*}=A\right\}
\end{aligned}
$$

of real and complex symmetric, real and complex skew-symmetric, and Hermitian matrices. We will use the simplified notation $\operatorname{Sym}(\mathbb{K})$, etc., when the size of the matrices is clear from the context. Our goal here is not just to give a sample mapping for each structure that realizes the minimal 2-norm, but to characterize the complete set of all minimal 2-norm mappings for each structure. For example, for real symmetric matrices it is already well known that $A=\left(\|b\|_{2} /\|x\|_{2}\right) H$, where $H$ is a Householder reflector mapping $x /\|x\|_{2}$ to $b /\|b\|_{2}$, provides a minimal 2-norm solution. However, the set of all minimal 2-norm symmetric matrices taking $x$ to $b$ has not previously been explicitly described.

First a preliminary lemma concerning the $2 \times 2$ case for a special type of $(x, b)$ vector pair.

Lemma 5.7. Let $\mathbb{S}$ be either $\operatorname{Sym}(2, \mathbb{K})$ or $\operatorname{Herm}(2, \mathbb{C})$ and

$$
\mathcal{S}=\left\{A \in \mathbb{S}: A\left[\begin{array}{l}
\alpha \\
\beta
\end{array}\right]=\left[\begin{array}{c}
\alpha \\
-\beta
\end{array}\right]\right\},
$$

where $\alpha, \beta \in \mathbb{C}$ with $\operatorname{Re}(\alpha) \neq 0$ and $\beta \neq 0$ when $\mathcal{S}=\operatorname{Sym}(2, \mathbb{C})$, and $\alpha, \beta \in \mathbb{R} \backslash\{0\}$ otherwise. Then

$$
\min _{A \in \mathcal{S}}\|A\|_{2}=1
$$

with $A=\left[\begin{array}{cc}1 & 0 \\ 0 & -1\end{array}\right]$ being the unique matrix in $\mathcal{S}$ of minimal 2-norm.

Proof. Note that from (5.6) any $A \in \mathcal{S}$ satisfies $\|A\|_{2} \geq 1$, and since $\left[\begin{array}{cc}1 & 0 \\ 0 & -1\end{array}\right] \in \mathcal{S}$ has unit 2-norm we have $\min _{A \in \mathcal{S}}\|A\|_{2}=1$. The rest of the proof consists of showing that $\left[\begin{array}{cc}1 & 0 \\ 0 & -1\end{array}\right]$ is the unique minimizer of the 2-norm for $\mathcal{S}$.

We start by parameterizing $\mathcal{S}$ using (4.1):

$$
\mathcal{S}=\left[\begin{array}{cc}
1 & 0 \\
0 & -1
\end{array}\right]+\left\{A \in \mathbb{S}: A\left[\begin{array}{l}
\alpha \\
\beta
\end{array}\right]=\left[\begin{array}{l}
0 \\
0
\end{array}\right]\right\}
$$

where $\left[\begin{array}{cc}1 & 0 \\ 0 & -1\end{array}\right]$ is a particular mapping in $\mathcal{S}$. Any $A \in \operatorname{Sym}(2, \mathbb{K})$ has the form $\left[\begin{array}{ll}a & z \\ z & c\end{array}\right]$ with $a, c, z \in \mathbb{K}$, so $A\left[\begin{array}{l}\alpha \\ \beta\end{array}\right]=\left[\begin{array}{l}0 \\ 0\end{array}\right]$ implies $\left[\begin{array}{ll}a & z \\ z & c\end{array}\right]=z\left[\begin{array}{cc}-\beta / \alpha & 1 \\ 1 & -\alpha / \beta\end{array}\right]$. Similarly any $A \in$ $\operatorname{Herm}(2, \mathbb{C})$ has the form $\left[\begin{array}{ll}a & z \\ \bar{z} & c\end{array}\right]$ with $a, c \in \mathbb{R}$; then $\alpha, \beta \in \mathbb{R}$ together with $A\left[\begin{array}{l}\alpha \\ \beta\end{array}\right]=\left[\begin{array}{l}0 \\ 0\end{array}\right]$ implies that $z \in \mathbb{R}$, and so $A$ can once again be expressed in the form $z\left[\begin{array}{cc}-\beta / \alpha & 1 \\ 1 & -\alpha / \beta\end{array}\right]$. Hence writing

$$
P(z)=\left[\begin{array}{cc}
1 & 0 \\
0 & -1
\end{array}\right]+z\left[\begin{array}{cc}
-\frac{\beta}{\alpha} & 1 \\
1 & -\frac{\alpha}{\beta}
\end{array}\right]=\left[\begin{array}{cc}
1-\frac{\beta}{\alpha} z & z \\
z & -1-\frac{\alpha}{\beta} z
\end{array}\right],
$$


we have $\mathcal{S}=\{P(z): \quad z \in \mathbb{K}\}$ if $\mathbb{S}=\operatorname{Sym}(2, \mathbb{K})$, and $\mathcal{S}=\{P(z): \quad z \in \mathbb{R}\}$ when $\mathbb{S}=\operatorname{Herm}(2, \mathbb{C})$ and $\alpha, \beta \in \mathbb{R}$.

We can now calculate the 2-norm of $P(z)$ by computing the largest eigenvalue of

$$
P^{*} P(z)=\left[\begin{array}{cc}
1-\left(\frac{\beta}{\bar{\alpha}} \bar{z}+\frac{\beta}{\alpha} z\right)+\left(1+\frac{\beta^{2}}{|\alpha|^{2}}\right)|z|^{2} & (z-\bar{z})-\gamma|z|^{2} \\
(\bar{z}-z)-\bar{\gamma}|z|^{2} & 1+\left(\frac{\bar{\alpha}}{\beta} \bar{z}+\frac{\alpha}{\beta} z\right)+\left(1+\frac{|\alpha|^{2}}{\beta^{2}}\right)|z|^{2}
\end{array}\right],
$$

where $\gamma:=(\alpha / \beta)+(\beta / \bar{\alpha})$. Much calculation and simplification yields

$$
\operatorname{tr} P^{*} P(z)=2+2 q(z), \quad \operatorname{det} P P^{*}(z)=1+2 q(z)-2\left(1+\operatorname{Re} \frac{\alpha^{2}}{|\alpha|^{2}}\right)|z|^{2},
$$

where $q(z):=\operatorname{Re}\left[\left(\frac{\alpha}{\beta}-\frac{\beta}{\alpha}\right) z\right]+|\gamma|^{2}|z|^{2} / 2 \in \mathbb{R}$. Since the characteristic polynomial of $P^{*} P(z)$ is $\lambda^{2}-\operatorname{tr} P^{*} P(z) \lambda+\operatorname{det} P^{*} P(z)$, we get

$$
\begin{aligned}
\lambda_{ \pm}(z) & =\frac{1}{2}\left(\operatorname{tr} P^{*} P(z) \pm \sqrt{\left(\operatorname{tr} P^{*} P(z)\right)^{2}-4 \operatorname{det} P^{*} P(z)}\right) \\
& =1+q(z) \pm \sqrt{q(z)^{2}+2\left(1+\operatorname{Re} \frac{\alpha^{2}}{|\alpha|^{2}}\right)|z|^{2}} .
\end{aligned}
$$

Since $q(z) \in \mathbb{R}$, clearly the largest eigenvalue of $P^{*} P(z)$ is $\lambda_{+}(z)$. But the hypothesis $\operatorname{Re}(\alpha) \neq 0$ means that $\operatorname{Re} \frac{\alpha^{2}}{|\alpha|^{2}}>-1$, so the second term under the square root is strictly bigger than 0 for all nonzero $z$. Hence $\lambda_{+}(z)$ satisfies $\lambda_{+}(0)=1$ and $\lambda_{+}(z)>1$ for all nonzero $z$. Thus $z=0$ is the unique minimizer of $\lambda_{+}(z)$, and hence $P(0)=\left[\begin{array}{cc}1 & 0 \\ 0 & -1\end{array}\right]$ is the unique minimizer of the 2-norm for $\mathcal{S}$. $\square$

We are now in a position to give a complete description of the set of all minimal 2-norm structured mappings for symmetric, skew-symmetric and Hermitian matrices. Recall that for nonzero $\mu \in \mathbb{K}$,

$$
\operatorname{sign}(\mu):=\bar{\mu} /|\mu| .
$$

Theorem 5.8 (Minimal 2-norm structured mappings: special cases). Let $\mathbb{S}_{n}$ be either $\operatorname{Sym}(n, \mathbb{K})$, Skew $(n, \mathbb{K})$, or $\operatorname{Herm}(n, \mathbb{C})$, and let $x, b \in \mathbb{K}^{n}$ be vectors such that $\mathcal{S}=\left\{A \in \mathbb{S}_{n}: A x=b\right\}$ is nonempty. Then

$$
\min _{A \in \mathcal{S}}\|A\|_{2}=\frac{\|b\|_{2}}{\|x\|_{2}}=: \rho .
$$

Furthermore there exists an $n \times n$ unitary matrix $U$ such that

$$
\mathcal{M}:=\left\{A \in \mathcal{S}:\|A\|_{2}=\rho\right\}=\left\{\rho U^{\star} \operatorname{diag}(R, S) U: S \in \mathbb{S}_{n-r},\|S\|_{2} \leq 1\right\},
$$

where the adjoint $\star$, the number $r$, and $R \in \mathbb{S}_{r}$ are given by:

(i) $\mathbb{S}_{n}=\operatorname{Sym}(n, \mathbb{K}): \star=T$ and $r=1(r=2)$ if $x$ and $\bar{b}$ are linearly dependent (independent), with

$$
R= \begin{cases}\operatorname{sign}(\mu) & \text { if } \bar{b}=\mu x \text { for some } \mu \in \mathbb{K}, \\
{\left[\begin{array}{ll}
1 & 0 \\
0 & -1
\end{array}\right]} & \text { otherwise, }\end{cases}
$$

(ii) $\mathbb{S}_{n}=\operatorname{Skew}(n, \mathbb{K}): \star=T$ and $r=2$, with $R=\left[\begin{array}{cc}0 & -1 \\ 1 & 0\end{array}\right]$, 
(iii) $\mathbb{S}_{n}=\operatorname{Herm}(n, \mathbb{C}): \star=*$ and $r=1(r=2)$ if $x$ and $b$ are linearly dependent (independent), with

$$
R= \begin{cases}\operatorname{sign}(\mu) & \text { if } b=\mu x \text { for some } \mu \in \mathbb{R}, \\
{\left[\begin{array}{cc}
1 & 0 \\
0-1
\end{array}\right]} & \text { otherwise. }\end{cases}
$$

The matrix $U$ can be taken as the product of at most two unitary ${ }^{1}$ Householder reflectors; when $\mathbb{K}=\mathbb{R}, U$ is real orthogonal.

Proof. For any $A \in \mathbb{S}_{n}$ such that $A x=b$, observe that the matrix $B=\rho^{-1} A$ is also in $\mathbb{S}_{n}$ and maps $\frac{x}{\|x\|_{2}}$ to $\frac{b}{\|b\|_{2}}$; also note that $\bar{b}=\mu x$ (resp., $b=\mu x$ ) in the theorem is equivalent to $\frac{\bar{b}}{\|b\|_{2}}=\operatorname{sign}(\bar{\mu}) \frac{x}{\|x\|_{2}}\left(\right.$ resp., $\left.\frac{b}{\|b\|_{2}}=\operatorname{sign}(\bar{\mu}) \frac{x}{\|x\|_{2}}\right)$. Thus it suffices to prove the theorem for $x, b \in \mathbb{K}^{n}$ with $\|x\|_{2}=\|b\|_{2}=1$ and the condition $\bar{b}=\mu x($ resp., $b=\mu x)$ replaced by $\bar{b}=\operatorname{sign}(\bar{\mu}) x($ resp., $b=\operatorname{sign}(\bar{\mu}) x)$; we make these assumptions throughout the rest of the argument.

The proof proceeds by first developing a unitary $U$ and accompanying $R$ for each of the five structures in (5.7). Then these $U$ and $R$ matrices are used to build explicit families of matrices in the structured mapping set $\mathcal{S}$ that realize the lower bound in (5.6), and thus are of minimal 2-norm. Finally we show that for each structure these families account for all of $\mathcal{M}$.

We begin by constructing for each case of the theorem a unitary matrix $U$ such that

$$
U x=\left[\begin{array}{l}
y \\
0
\end{array}\right], \quad\left(U^{\star}\right)^{-1} b=\left[\begin{array}{l}
c \\
0
\end{array}\right],
$$

with $y, c \in \mathbb{K}^{r}$ satisfying $R y=c$, where $R \in \mathbb{S}_{r}$ is as defined in the theorem.

(i) First, suppose that $\mathbb{S}_{n}=\operatorname{Sym}(n, \mathbb{K})$. If $\bar{b}=\operatorname{sign}(\bar{\mu}) x$ for some $\mu \in \mathbb{K}$, then let $U$ be the unitary Householder reflector mapping $x$ to $e_{1}$, so that $y=1$. Then $\left(U^{\star}\right)^{-1} b=\bar{U} b=\operatorname{sign}(\mu) e_{1}$, so $c=\operatorname{sign}(\mu)$. Clearly with $R:=\operatorname{sign}(\mu) \in \mathbb{S}_{1}$ we have $R y=c$.

When $x$ and $\bar{b}$ are linearly independent then $U$ can be taken as the product of two unitary Householder reflectors, $U=H_{2} H_{1}$. The first reflector $H_{1}$ takes $x+\bar{b}$ to $\pm\|x+\bar{b}\|_{2} e_{1}$; with $H_{1} x=\left[\begin{array}{l}\alpha \\ v\end{array}\right]$ and $H_{1} \bar{b}=\left[\begin{array}{c}\gamma \\ w\end{array}\right]$ we see that $w=-v$ with $v \neq 0$ because of the linear independence of $x$ and $\bar{b}$, and $\alpha+\gamma= \pm\|x+\bar{b}\|_{2} \in \mathbb{R} \backslash\{0\}$. Then $\|x\|_{2}=\|\bar{b}\|_{2} \Rightarrow\left\|H_{1} x\right\|_{2}=\left\|H_{1} \bar{b}\right\|_{2} \Rightarrow|\alpha|=|\gamma|$, which together with $\alpha+\gamma \in \mathbb{R}$ implies that $\gamma=\bar{\alpha}$, and hence $H_{1} \bar{b}=\left[\begin{array}{c}\bar{\alpha} \\ -v\end{array}\right]$. Note also that $2 \operatorname{Re} \alpha=\alpha+\bar{\alpha}= \pm\|x+\bar{b}\|_{2} \neq 0$. For the second reflector pick $H_{2}=\left[\begin{array}{cc}1 & 0 \\ 0 & \widetilde{H}_{2}\end{array}\right]$ so that $\widetilde{H}_{2} v=\beta e_{1}$ with $\beta= \pm\|v\|_{2} \neq 0$. Hence

$$
U\left[\begin{array}{ll}
x & \bar{b}
\end{array}\right]=\left[\begin{array}{cc}
\alpha & \bar{\alpha} \\
\beta & -\beta \\
0 & 0 \\
\vdots & \vdots \\
0 & 0
\end{array}\right], \quad \operatorname{Re} \alpha \neq 0,0 \neq \beta \in \mathbb{R},
$$

and therefore $y=\left[\begin{array}{l}\alpha \\ \beta\end{array}\right]$ and $c=\left[\begin{array}{c}\alpha \\ -\beta\end{array}\right]$ satisfy $R y=c$ with $R=\left[\begin{array}{cc}1 & 0 \\ 0 & -1\end{array}\right] \in \mathbb{S}_{2}$. Note that $U$ can be taken to be real orthogonal when $\mathbb{K}=\mathbb{R}$.

\footnotetext{
${ }^{1}$ but not necessarily Hermitian, see [12].
} 
(ii) For $\mathbb{S}_{n}=\operatorname{Skew}(n, \mathbb{K})$, Theorem 3.2 says that $\mathcal{S}$ is nonempty if and only if $b^{T} x=0$. In this situation $U$ can be taken as the product of two unitary Householder reflectors, $U=H_{2} H_{1}$. The first reflector $H_{1}$ is defined to take $x$ to $e_{1}$; then $H_{1} \bar{b}=\left[\begin{array}{c}\alpha \\ v\end{array}\right]$ for some $v \in \mathbb{K}^{n-1}$. The fact that $b^{T} x=0$ implies $\alpha=0$, since $b^{T} x=\left[\begin{array}{ll}\bar{\alpha} & v^{*}\end{array}\right] H_{1} H_{1}^{*} e_{1}=\bar{\alpha}$. For the second reflector pick $H_{2}=\left[\begin{array}{cc}1 & 0 \\ 0 & \widetilde{H}_{2}\end{array}\right]$ so that $\widetilde{H}_{2} v=e_{1} \in \mathbb{K}^{n-1}$. Then $U\left[\begin{array}{ll}x & \bar{b}\end{array}\right]=\left[\begin{array}{ll}e_{1} & e_{2}\end{array}\right] \in \mathbb{K}^{n \times 2}$, giving $y=\left[\begin{array}{l}1 \\ 0\end{array}\right]$ and $c=\left[\begin{array}{l}0 \\ 1\end{array}\right]$ in $\mathbb{K}^{2}$ satisfying $R y=c$ for $R=\left[\begin{array}{cc}0 & -1 \\ 1 & 0\end{array}\right] \in \mathbb{S}_{2}$. Note once again that $U$ can be taken to be real orthogonal when $\mathbb{K}=\mathbb{R}$.

(iii) Finally suppose that $\mathbb{S}_{n}=\operatorname{Herm}(n, \mathbb{C})$. Theorem 3.2 says that $\mathcal{S}$ is nonempty if and only if $b^{*} x \in \mathbb{R}$. If $x$ and $b$ are linearly dependent, then $b=\operatorname{sign}(\bar{\mu}) x$ for some $\mu \in \mathbb{C}$, and $b^{*} x \in \mathbb{R}$ implies that $\mu \in \mathbb{R}$. In this case $U$ can be taken as the unitary Householder reflector mapping $x$ to $e_{1}$ so that $\left(U^{\star}\right)^{-1} b=U b=\operatorname{sign}(\mu) e_{1}$ since $\mu$ is real. Hence $\left[\begin{array}{ll}y & c\end{array}\right]=\left[\begin{array}{ll}1 & \operatorname{sign}(\mu)\end{array}\right]$ and $R y=c$ with $R=\operatorname{sign}(\mu) \in \mathbb{S}_{1}$.

On the other hand if $x$ and $b$ are linearly independent, then $U$ can be taken as the product of two unitary Householder reflectors $U=H_{2} H_{1}$ in a manner analogous to that described above in (i) for $\operatorname{Sym}(n, \mathbb{K})$; the only difference is that $H_{1}$ now takes $x+b$ to $\pm\|x+b\|_{2} e_{1}$. In this case (5.9) holds with $\bar{b}$ replaced by $b$. Also $b^{*} x=\left(H_{1} b\right)^{*}\left(H_{1} x\right)=\alpha^{2}-v^{*} v \in \mathbb{R}$ so that $\alpha^{2} \in \mathbb{R}$. This together with $\operatorname{Re} \alpha \neq 0$ implies that $\alpha \in \mathbb{R}$. Hence we have $y=\left[\begin{array}{l}\alpha \\ \beta\end{array}\right]$ and $c=\left[\begin{array}{c}\alpha \\ -\beta\end{array}\right]$ with $\alpha, \beta \in \mathbb{R} \backslash\{0\}$, satisfying $R y=c$ with $R=\left[\begin{array}{cc}1 & 0 \\ 0 & -1\end{array}\right] \in \mathbb{S}_{2}$.

Using the unitary $U$ and $R \in \mathbb{S}_{r}$ constructed above for each $\mathbb{S}_{n}$, we can now show that the lower bound $1=\|b\|_{2} /\|x\|_{2} \leq \min _{A \in \mathcal{S}}\|A\|_{2}$ from (5.6) is actually attained by a whole family of $A \in \mathcal{S}$. For any $S \in \mathbb{S}_{n-r}$ with $\|S\|_{2} \leq 1$, consider $A=U^{\star} \operatorname{diag}(R, S) U$. Then $A \in \mathbb{S}_{n}$, since $\mathbb{S}_{n}$ is preserved by any $\star$-congruence (see Lemma 4.1(a)) and $\operatorname{diag}(R, S) \in \mathbb{S}_{n}$. Also $A x=b$ because of the properties of $U$ in (5.8), and $\|A\|_{2}=\|\operatorname{diag}(R, S)\|_{2}=\|R\|_{2}=1$. Thus

$$
\left\{U^{\star} \operatorname{diag}(R, S) U: S \in \mathbb{S}_{n-r},\|S\|_{2} \leq 1\right\} \subseteq \mathcal{M}
$$

Finally, we complete the characterization of $\mathcal{M}$ by showing that this containment is actually an equality. Consider an arbitrary $A \in \mathcal{M}$. Then $A x=b \Rightarrow$ $\left(\left(U^{\star}\right)^{-1} A U^{-1}\right)(U x)=\left(U^{\star}\right)^{-1} b$, so the matrix $B:=\left(U^{\star}\right)^{-1} A U^{-1}=\left(U^{-1}\right)^{\star} A U^{-1}$ is in $\mathbb{S}_{n}$ and maps the vector $U x=\left[\begin{array}{l}y \\ 0\end{array}\right]$ to $\left(U^{\star}\right)^{-1} b=\left[\begin{array}{l}c \\ 0\end{array}\right]$. Let $B_{11} \in \mathbb{S}_{r}$ be the leading principal $r \times r$ submatrix of $B$, so $\left\|B_{11}\right\|_{2} \leq\|B\|_{2}=\|A\|_{2}=1$. The form of the two vectors $\left[\begin{array}{l}y \\ 0\end{array}\right]$ and $\left[\begin{array}{l}c \\ 0\end{array}\right]$ implies that $B_{11}$ maps $y$ to $c$; since $\|y\|_{2}=\|c\|_{2}=1$ we have $\left\|B_{11}\right\|_{2} \geq 1$, and hence $\left\|B_{11}\right\|_{2}=1$. Using Lemma 5.7 we can now show that $B_{11}=R$ in all cases.

(i) Suppose $\mathbb{S}_{n}=\operatorname{Sym}(n, \mathbb{K})$ and $B_{11} \in \mathbb{S}_{r}$. Then $\bar{b}=\operatorname{sign}(\bar{\mu}) x$ for some $\mu \in \mathbb{K}$ implies $[y c]=[1 \operatorname{sign}(\mu)]$, so $B_{11} y=c$ implies $B_{11}=\operatorname{sign}(\mu)=R$. On the other

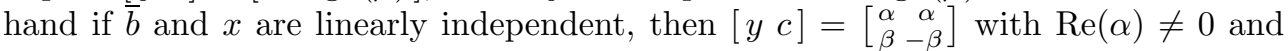
$0 \neq \beta \in \mathbb{R}$. Since $B_{11} y=c$ with $\left\|B_{11}\right\|_{2}=1$, Lemma 5.7 implies that $B_{11}=\left[\begin{array}{cc}1 & 0 \\ 0 & -1\end{array}\right]=$ $R$.

(ii) $B_{11} \in \operatorname{Skew}(2, \mathbb{K})$ must have the form $\left[\begin{array}{cc}0 & -\sigma \\ \sigma & 0\end{array}\right]$ for some $\sigma \in \mathbb{K}$. So $B_{11} y=c$ with $y=e_{1}$ and $c=e_{2}$ implies $\sigma=1$, and hence $B_{11}=\left[\begin{array}{cc}0 & -1 \\ 1 & 0\end{array}\right]=R$.

(iii) Finally consider $\mathbb{S}_{n}=\operatorname{Herm}(n, \mathbb{C})$ and $B_{11} \in \mathbb{S}_{r}$. If $b=\operatorname{sign}(\mu) x$ for some $\mu \in \mathbb{R}$ then $[y c]=[1 \operatorname{sign}(\mu)]$, so $B_{11} y=c$ implies $B_{11}=\operatorname{sign}(\mu)=R$. If $b$ and $x$

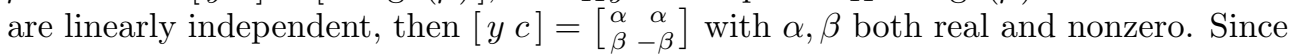
$B_{11} y=c$ with $\left\|B_{11}\right\|_{2}=1$, Lemma 5.7 implies that $B_{11}=\left[\begin{array}{cc}1 & 0 \\ 0 & -1\end{array}\right]=R$. 
The condition $\|B\|_{2}=1$ now forces the rest of the first $r$ columns of $B$ to be all zeroes; since $B \in \mathbb{S}_{n}$, the rest of the first $r$ rows of $B$ must also be all zeroes. Thus $B$ has the form $B=\operatorname{diag}(R, S)$, with $S \in \mathbb{S}_{n-r}$. Finally, $\|B\|_{2}=1$ and $\|R\|_{2}=1$ implies that $\|S\|_{2} \leq 1$. Thus we have $B:=\left(U^{\star}\right)^{-1} A U^{-1}=\operatorname{diag}(R, S)$, so $A=U^{\star} \operatorname{diag}(R, S) U$ and hence $\mathcal{M} \subseteq\left\{U^{\star} \operatorname{diag}(R, S) U: S \in \mathbb{S}_{n-r},\|S\|_{2} \leq 1\right\}$.

5.3.2. More general $\mathbb{L}$ and $\mathbb{J}$. For Lie algebras $\mathbb{L}$ and Jordan algebras $\mathbb{J}$ of scalar products $\langle\cdot, \cdot\rangle_{\mathrm{M}}$ that are both unitary and orthosymmetric, the minimal 2norm structured mapping problem can be reduced to one of the five cases treated in section 5.3.1. To this end we first show that for orthosymmetric $\langle\cdot, \cdot\rangle_{\mathrm{M}}$, left multiplication by the matrix $M$ defining the scalar product is a bijection from $\mathbb{K}^{n \times n}$ to $\mathbb{K}^{n \times n}$ that maps $\mathbb{L}$ and $\mathbb{J}$ to $\operatorname{Skew}(\mathbb{K})$ and $\operatorname{Sym}(\mathbb{K})$ for bilinear forms, and to scalar multiples of $\operatorname{Herm}(\mathbb{C})$ for sesquilinear forms. This result appeared in [13, Thm. 8.4], but we repeat the proof here for the convenience of the reader. The reduction to the five special cases $\operatorname{Sym}(\mathbb{K}), \operatorname{Skew}(\mathbb{K})$, and $\operatorname{Herm}(\mathbb{C})$ is then achieved by the 2-normpreserving bijection $A \mapsto \widetilde{A}:=\beta M A$, where $\beta>0$ is a real constant such that $\beta M$ is unitary.

LEMмa 5.9. Let $\mathbb{S}$ be the Lie or Jordan algebra of an orthosymmetric scalar product $\langle\cdot, \cdot\rangle_{\mathrm{M}}$. Suppose $A \in \mathbb{S}$, so that $A^{\star}=\delta A$ where $\delta= \pm 1$.

- For bilinear forms on $\mathbb{K}^{n}$ write, by orthosymmetry, $M=\alpha M^{T}$ with $\alpha= \pm 1$. Then

$$
M \cdot \mathbb{S}= \begin{cases}\operatorname{Sym}(\mathbb{K}) & \text { if } \delta=\alpha \\ \operatorname{Skew}(\mathbb{K}) & \text { if } \delta \neq \alpha .\end{cases}
$$

- For sesquilinear forms on $\mathbb{C}^{n}$ write, by orthosymmetry, $M=\alpha M^{*}$ with $|\alpha|=1$. Then

$$
M \cdot \mathbb{S}= \begin{cases}\alpha^{1 / 2} \operatorname{Herm}(\mathbb{C}) & \text { if } \delta=+1 \\ \alpha^{1 / 2} i \operatorname{Herm}(\mathbb{C}) & \text { if } \delta=-1\end{cases}
$$

Proof. For orthosymmetric bilinear forms

$$
A \in \mathbb{S} \Longrightarrow \delta A=A^{\star}=M^{-1} A^{T} M \Longrightarrow \delta M A=A^{T} M=\alpha(M A)^{T},
$$

which shows that $M A$ is (complex) symmetric if $\delta=\alpha$ and (complex) skew-symmetric if $\delta \neq \alpha$. Combining this with the property $\mathbb{K}^{n \times n}=\mathbb{L} \oplus \mathbb{J}$ for orthosymmetric scalar products (see sec. 2.4) completes the proof of (5.10).

Similarly, for orthosymmetric sesquilinear forms we have

$$
A \in \mathbb{S} \Leftrightarrow \delta A=A^{\star}=M^{-1} A^{*} M \Leftrightarrow \delta M A=A^{*} M \Leftrightarrow \delta \bar{\alpha}^{1 / 2} M A=\left(\bar{\alpha}^{1 / 2} M A\right)^{*} .
$$

Hence $\bar{\alpha}^{1 / 2} M A$ is either Hermitian or skew-Hermitian. Together with $\mathbb{C}^{n \times n}=\mathbb{L} \oplus \mathbb{J}$ this proves (5.11).

We are now in a position to prove the main result of section 5.3.

THEOREM 5.10 (Minimal 2-norm structured mappings: general case). Let $\mathbb{S}_{n}$ be the Lie algebra $\mathbb{L}$ or Jordan algebra $\mathbb{J}$ of a scalar product $\langle\cdot, \cdot\rangle_{\mathrm{M}}$ on $\mathbb{K}^{n}$ that is both orthosymmetric and unitary, so that $M \cdot \mathbb{S}_{n}$ is either $\operatorname{Sym}(n, \mathbb{K})$, Skew $(n, \mathbb{K})$, or $\gamma \operatorname{Herm}(n, \mathbb{C})$ for some $|\gamma|=1$. Also let $x, b \in \mathbb{K}^{n}$ be vectors such that $\mathcal{S}=\left\{A \in \mathbb{S}_{n}\right.$ : $A x=b\}$ is nonempty. Then

$$
\min _{A \in \mathcal{S}}\|A\|_{2}=\frac{\|b\|_{2}}{\|x\|_{2}}:=\rho .
$$


Furthermore there exists a unitary matrix $U$ such that

$$
\mathcal{M}:=\left\{A \in \mathcal{S}:\|A\|_{2}=\rho\right\}=\left\{\rho U^{\star}(\beta M)^{-1} \operatorname{diag}(R, S) U: S \in \widetilde{\mathbb{S}}_{n-r},\|S\|_{2} \leq 1\right\},
$$

where $\beta>0$ is a real constant such that $\beta M$ is unitary, $\star$ denotes the adjoint of the scalar product $\langle\cdot, \cdot\rangle_{\mathrm{M}}$, and the number $r$, the structured class $\widetilde{\mathbb{S}}_{n-r}$, and $R \in \mathbb{K}^{r \times r}$ are given in each case by:

(i) $M \cdot \mathbb{S}_{n}=\operatorname{Sym}(n, \mathbb{K}): r=1(r=2)$ if $x$ and $\overline{M b}$ are linearly dependent (independent $), \widetilde{\mathbb{S}}_{n-r}=\operatorname{Sym}(n-r, \mathbb{K})$, and

$$
R= \begin{cases}\operatorname{sign}(\mu) & \text { if } \beta \overline{M b}=\mu x \text { for some } \mu \in \mathbb{K}, \\
{\left[\begin{array}{cc}
1 & 0 \\
0 & -1
\end{array}\right]} & \text { otherwise, }\end{cases}
$$

(ii) $M \cdot \mathbb{S}_{n}=\operatorname{Skew}(n, \mathbb{K}): r=2, \widetilde{\mathbb{S}}_{n-r}=\operatorname{Skew}(n-2, \mathbb{K})$, and $R=\left[\begin{array}{cc}0 & -1 \\ 1 & 0\end{array}\right]$,

(iii) $M \cdot \mathbb{S}_{n}=\gamma \operatorname{Herm}(n, \mathbb{C})$ for some $|\gamma|=1: \quad r=1(r=2)$ if $x$ and $M b$ are linearly dependent $($ independent $), \widetilde{\mathbb{S}}_{n-r}=\gamma \operatorname{Herm}(n-r, \mathbb{C})$, and

$$
R= \begin{cases}\gamma \operatorname{sign}(\mu) & \text { if } \gamma^{-1} \beta M b=\mu x \text { for some } \mu \in \mathbb{R}, \\
\gamma\left[\begin{array}{cc}
1 & 0 \\
0 & -1
\end{array}\right] & \text { otherwise. }\end{cases}
$$

The matrix $U$ can be taken as the product of at most two unitary Householder reflectors; when $\mathbb{K}=\mathbb{R}, U$ is real orthogonal.

Proof. Lemma 5.9 together with $\beta M$ being unitary implies that left multiplication by $\beta M$ is a 2-norm-preserving bijection from $\mathbb{K}^{n \times n}$ to $\mathbb{K}^{n \times n}$ that maps $\mathbb{S}_{n}$ to $\operatorname{Skew}(n, \mathbb{K})$ or $\operatorname{Sym}(n, \mathbb{K})$ for bilinear forms, and $\mathbb{S}_{n}$ to $\gamma \operatorname{Herm}(n, \mathbb{C})$ for sesquilinear forms. For bilinear forms, the equivalence of the equations $A x=b$ and

$$
\widetilde{A} x:=(\beta M A) x=(\beta M b)=: \widetilde{b}
$$

thus reduces the structured mapping problem for $\mathcal{S}=\left\{A \in \mathbb{S}_{n}: A x=b\right\}$ in a 2norm-preserving way to the structured mapping problem for finding $\widetilde{A}$ in $\operatorname{Skew}(n, \mathbb{K})$ or $\operatorname{Sym}(n, \mathbb{K})$ such that $\widetilde{A} x=\widetilde{b}$. Similarly for sesquilinear forms, the equivalence of $A x=b$ and $\widetilde{A} x:=\left(\gamma^{-1} \beta M A\right) x=\left(\gamma^{-1} \beta M b\right)=: \widetilde{b}$ gives a 2-norm-preserving reduction of the structured mapping problem for $\mathcal{S}$ to that of finding $\widetilde{A}$ in $\operatorname{Herm}(n, \mathbb{C})$ such that $\widetilde{A} x=\widetilde{b}$.

The value of $\min _{A \in \mathcal{S}}\|A\|_{2}$ and the formula for $\mathcal{M}$ now follow by applying Theorem 5.8 to the minimal 2 -norm structured mapping problem for $\widetilde{A} x=\widetilde{b}$, and then using the correspondence between $\widetilde{A}$ and $A$.

5.4. Comparison of the various "minimal" structured mappings. We conclude section 5 by exploring the relationships between the three types of extremal mappings - minimal rank, Frobenius norm, and 2-norm - under the assumption that the scalar product $\langle\cdot, \cdot\rangle_{\mathrm{M}}$ is both unitary and orthosymmetric.

In general the minimal Frobenius norm solution $A_{\mathrm{opt}}$ differs from the minimal rank solution. The latter is usually rank one whereas $A_{\mathrm{opt}}$ is generally rank-two. From (5.2) we see that $A_{\text {opt }}$ is rank-one if and only if $M^{-1} \bar{x} \in \operatorname{span}\{b\}$ for bilinear forms or $M^{-1} x \in \operatorname{span}\{b\}$ for sesquilinear forms.

For structured mappings of minimal 2-norm, the following corollary of Theorem 5.10 singles out the unique matrix of minimal rank as well as the one of minimal Frobenius norm. 
Corollary 5.11. Under the hypotheses of Theorem 5.10, let $\mathcal{M}$ denote the set of all minimal 2-norm mappings in $\mathcal{S}=\{A \in \mathbb{S}: A x=b\}$. Assume further that $x, b$ are vectors such that $\mathcal{S}$ is nonempty. Consider the particular mapping

$$
A_{2}:=\frac{\|b\|_{2}}{\|x\|_{2}} U^{\star}(\beta M)^{-1}\left[\begin{array}{cc}
R & 0 \\
0 & 0
\end{array}\right] U \in \mathcal{M}
$$

obtained by setting $S$ equal to 0 in (5.12). Then $A_{2}$ is the unique solution of both the minimal rank problem $\min \operatorname{rank}(A)$ and the minimal Frobenius norm problem $\min _{A \in \mathcal{M}}\|A\|_{F}$. Moreover, either

(1) $A_{2}$ has rank 1 and $\left\|A_{2}\right\|_{F}=\|b\|_{2} /\|x\|_{2}$, or

(2) $A_{2}$ has rank 2 and $\left\|A_{2}\right\|_{F}=\sqrt{2}\|b\|_{2} /\|x\|_{2}$.

Case (1) occurs when $x$ and $\overline{M b}$ ( $x$ and $M b)$ are linearly dependent and the scalar product is bilinear (sesquilinear). (Note that if $M \cdot \mathbb{S}=\operatorname{Skew}(n, \mathbb{K})$, then this linear dependence implies that $\mathcal{S}$ is empty.) Otherwise case (2) holds.

Are there any conditions under which there is a structured mapping in $\mathcal{S}$ that simultaneously has all three extremal properties? The next result provides a complete answer to this question.

THEOREM 5.12. Let $\mathbb{S}$ be the Lie or Jordan algebra of a scalar product $\langle\cdot, \cdot\rangle_{M}$ that is both unitary and orthosymmetric. Assume that $x, b \in \mathbb{K}^{n} \backslash\{0\}$ are vectors such that $\mathcal{S}=\{A \in \mathbb{S}: A x=b\}$ is nonempty. Then the unique minimal Frobenius norm mapping $A_{\mathrm{opt}} \in \mathcal{S}$ has both minimal 2-norm and minimal rank in $\mathcal{S}$ if and only if the pair of vectors $(x, b)$ satisfies either property (a) or property (b) below.

(a) $M^{-1} \bar{x} \in \operatorname{span}\{b\}$ for bilinear forms or $M^{-1} x \in \operatorname{span}\{b\}$ for sesquilinear forms. In this case

$$
A_{\text {opt }}=A_{2}= \begin{cases}b b^{T} M /\langle b, x\rangle_{\mathrm{M}} & \text { for bilinear forms }, \\ b b^{*} M /\langle b, x\rangle_{\mathrm{M}} & \text { for sesquilinear forms }\end{cases}
$$

is the unique rank-one mapping in $\mathcal{S}$.

(b) $\langle b, x\rangle_{M}=0$. In this case

$$
A_{\mathrm{opt}}=A_{2}=\frac{b x^{*}}{x^{*} x}+\epsilon\left(\frac{b x^{*}}{x^{*} x}\right)^{\star}, \quad \epsilon= \begin{cases}1 & \text { if } \mathbb{S}=\mathbb{J} \\ -1 & \text { if } \mathbb{S}=\mathbb{L}\end{cases}
$$

is the unique rank-two mapping in $\mathcal{M}=\left\{A \in \mathcal{S}:\|A\|_{2}=\min _{B \in \mathcal{S}}\|B\|_{2}\right\}$.

Proof. $(\Rightarrow) A_{\text {opt }}$ having minimal 2-norm in $\mathcal{S}$ means that $A_{\text {opt }} \in \mathcal{M}$, with minimal Frobenius norm in $\mathcal{M}$; thus $A_{\text {opt }}=A_{2}$ by Corollary 5.11. But $A_{2}$ is either rank 1 or rank $2 . A_{2}$ with rank 1 means $A_{\text {opt }}$ has rank 1 , and therefore property (a) holds by the remarks preceding the corollary. On the other hand $A_{2}$ with rank 2 implies $\left\|A_{\text {opt }}\right\|_{F}=\left\|A_{2}\right\|_{F}=\sqrt{2}\|b\|_{2} /\|x\|_{2}$, which by (5.3) implies that property (b) holds.

$(\Leftarrow)$ Property (a) implies that $A_{\text {opt }}$ is rank 1 by the remarks preceding the corollary. But property (a) is equivalent to the linear dependence of $x$ and $\overline{M b}(x$ and $M b)$ for bilinear (sesquilinear) forms, which are precisely the conditions in Corollary 5.11 which guarantee that $A_{2}$ is rank 1 . The uniqueness of rank-one mappings in $\mathcal{S}$ from Theorem 5.1 now implies that $A_{\mathrm{opt}}=A_{2}$ has all three minimality properties.

Property (b) implies that $\left\|A_{\mathrm{opt}}\right\|_{F}=\sqrt{2}\|b\|_{2} /\|x\|_{2}$ by (5.3), and that the minimal rank in $\mathcal{S}$ is 2 by Theorem 5.3. By Corollary 5.11 we know that $\left\|A_{2}\right\|_{F} \leq$ $\sqrt{2}\|b\|_{2} /\|x\|_{2}$, so the uniqueness of minimal Frobenius norm mappings implies that $A_{\text {opt }}=A_{2}$. This map has minimal rank 2 by case (2) of Corollary 5.11 . 
6. Concluding remarks. A complete, unified solution for the existence and characterization part of the structured mapping problems for Lie and Jordan algebras of orthosymmetric scalar products has been presented. Amongst all structured mappings, solutions of minimal rank were identified and a condition on $x$ and $b$ for uniqueness was given. The minimal Frobenius norm structured solution was shown to be unique and an expression for it obtained when the scalar product is both orthosymmetric and unitary. A characterization of the set of all minimal 2-norm structured mappings was also presented.

It will be interesting to extend our results to the case of multiple right-hand sides.

Associated with a scalar product $\langle\cdot, \cdot\rangle_{\mathrm{M}}$ is a third class of structured matrices: the automorphism group $\mathbb{G}$ defined by

$$
\mathbb{G}=\left\{A \in \mathbb{K}^{n \times n}:\langle A x, A y\rangle_{\mathrm{M}}=\langle x, y\rangle_{\mathrm{M}} \forall x, y \in \mathbb{K}^{n}\right\}=\left\{A \in \mathbb{K}^{n \times n}: A^{\star}=A^{-1}\right\} .
$$

Unlike the corresponding Jordan and Lie algebras, $\mathbb{G}$ is not a linear subspace of $\mathbb{K}^{n \times n}$ : it is a multiplicative group and the structure is nonlinear. Examples of automorphism groups include the orthogonal, symplectic, and pseudo-unitary groups. The existence and characterization problems for automorphism groups are addressed in part II of this paper [11]. There it is shown that for any orthosymmetric scalar product $\langle\cdot, \cdot\rangle_{\mathrm{M}}$ there exists $A \in \mathbb{G}$ such that $A x=b$ if and only if $\langle x, x\rangle_{\mathrm{M}}=\langle b, b\rangle_{\mathrm{M}}$. Two characterizations of the set $\{A \in \mathbb{G}: A x=b\}$ of all structured mappings are proposed: one multiplicative, the other additive. We expect both to be useful for backward error investigations.

\section{REFERENCES}

[1] J. M. Borwein AND A. S. Lewis, Convex analysis and nonlinear optimization. Theory and examples, Springer-Verlag, New York, 2000.

[2] J. R. Bunch, J. W. Demmel, and C. F. VAN LOAN, The strong stability of algorithms for solving symmetric linear systems, SIAM J. Matrix Anal. Appl., 10 (1989), pp. 494-499.

[3] J. E. Dennis, JR. And J. J. Moré, Quasi-Newton methods, motivation and theory, SIAM Rev., 19 (1977), pp. 46-89.

[4] D. J. Higham And N. J. Higham, Backward error and condition of structured linear systems, SIAM J. Matrix Anal. Appl., 13 (1992), pp. 162-175.

[5] R. A. Horn, V. V. Sergeichuk, and N. Shaked-Monderer, Solution of linear matrix equations in $a{ }^{*}$ congruence class, Electron. J. Linear Algebra, 13 (2005), pp. 153-156.

[6] C. R. Johnson And R. L. Smith, Linear interpolation problems for matrix classes and a transformational characterization of $M$-matrices, Linear Algebra Appl., 330 (2001), pp. 43-48.

[7] M. Karow, D. Kressner, and F. Tisseur, Structured eigenvalue condition numbers, Numerical Analysis Report No. 467, Manchester Centre for Computational Mathematics, Manchester, England, Apr. 2005.

[8] C. Khatri and S. K. Mitra, Hermitian and nonnegative definite solutions of linear matrix equations., SIAM J. Appl. Math., 31 (1976), pp. 579-585.

[9] R. LIU AND R. LEAKE, Exhaustive equivalence classes of optimal systems with separable controls, SIAM J. Control, 4 (1966), pp. 678-685.

[10] M. Machover, Matrices which take a given vector into a given vector, Amer. Math. Monthly, 74 (1967), pp. 851-852.

[11] D. S. Mackey, N. Mackey, and F. Tisseur, Structured mapping problems for matrices associated with scalar products part II: Automorphism groups, MIMS EPrint, Manchester Institute for Mathematical Sciences, The University of Manchester, UK. In preparation.

[12] — G-reflectors: Analogues of Householder transformations in scalar product spaces, Linear Algebra Appl., 385 (2004), pp. 187-213.

[13] _ - Structured factorizations in scalar product spaces, SIAM J. Matrix Anal. Appl., 27 (2006), pp. 821-850.

[14] A. Pinkus, Interpolation by matrices, Electron. J. Linear Algebra, 11 (2004), pp. 281-291.

[15] S. M. RumP, Structured perturbations part I: Normwise distances, SIAM J. Matrix Anal. Appl., 25 (2003), pp. 1-30. 
[16] J. Sun, Backward perturbation analysis of certain characteristic subspaces, Numer. Math., 65 (1993), pp. 357-382.

[17] - Backward errors for the unitary eigenproblem, Tech. Report UMINF-97.25, Department of Computing Science, University of Umeå, Umeå, Sweden, 1997.

[18] F. Tisseur, A chart of backward errors and condition numbers for singly and doubly structured eigenvalue problems, SIAM J. Matrix Anal. Appl., 24 (2003), pp. 877-897.

[19] F. Tisseur and S. Graillat, Structured condition numbers and backward errors in scalar product spaces, MIMS EPrint 2006.16, Manchester Institute for Mathematical Sciences, The University of Manchester, UK, Feb. 2006.

[20] G. TRenkleR, Matrices which take a given vector into a given vector-revisited, Amer. Math. Monthly, 111 (2004), pp. 50-52.

[21] Z.-Z. ZhANG, X.-Y. Hu, AND L. ZhANG, On the Hermitian-generalized Hamiltonian solutions of linear matrix equations, SIAM J. Matrix Anal. Appl., 27 (2005), pp. 294-303 (electronic). 